\title{
A DIFFERENCE METHOD FOR THE MCKEAN-VLASOV EQUATION
}

\author{
G. M. COCLITE AND N. H. RISEBRO
}

\begin{abstract}
We analyze a model equation arising in option pricing. This model equation takes the form of a non-linear, non-local diffusion equation. We prove the well-posedness of the Cauchy problem for this equation. Furthermore, we introduce a semidiscrete difference scheme and show its rate of convergence.
\end{abstract}

\section{INTRODUCTION}

A huge amount of option pricing models has been developed since the seminal work of Black, Scholes and Merton [2], who model the evolution of an underlying (e.g. stock) as a geometric Brownian motion with drift. One branch of literature focuses on local volatility models, which extend the Black-Scholes model by the introduction of a spatially (and temporaly) inhomogeneous volatility parameter. It is well-known that these models allow for a perfect fit under weak assumptions on the data. A drawback of such models is their inability to produce realistic paths of the underlying, and unrealistic parameters in certain situations. Another branch of literatures focuses on stochastic volatility models, such as the Hull-White model, the Heston model or the SABR model, see $[6,7]$. These models assume a stochastic evolution of the volatility variable. Such models often lead to more realistic pricing paths, but usually do not allow for a perfect fit to data, due to a small number of degrees of freedom. More recently, [5] introduced a combination of both models, so-called local-stochastic volatility models, which combine the advantages of both approaches.

This paper is dedicated to the well-posedness and convergence of a numerical scheme fo the Cauchy problem

$$
\begin{cases}\partial_{t} u=\Delta_{x}(\mathcal{I}[u] u)+\Delta_{y} u, & t>0, x \in \mathbb{R}^{M}, y \in \mathbb{R}^{N}, \\ u(0, x, y)=u_{0}(x, y), & x \in \mathbb{R}^{M}, y \in \mathbb{R}^{N}\end{cases}
$$

where

$$
\mathcal{I}[u](x)=\int_{\mathbb{R}^{M}} \int_{\mathbb{R}^{N}} b(x, \xi, z) u(\xi, z) d \xi d z, \quad x \in \mathbb{R}^{M} .
$$

Here $M \geq 1$, and $N \geq 0$, if $N=0$ the last term with $\Delta_{y}$ is absent.

Pricing of European options in the described models leads to different type of partial differential equations (see [3]). While option prices in the Black-Scholes model correspond to solutions of a univariate parabolic PDE with constant coefficients, option pricing in local volatility framework leads to the solution of parabolic PDEs with non-constant and non-degenerate diffusion coefficients $\sigma$ such as

$$
\left\{\begin{array}{l}
\partial_{t} u-\partial_{x}\left(\sigma(x) \partial_{x} u\right)=0 \\
u(0, \cdot)=u_{0}
\end{array}\right.
$$

Date: September 4, 2019.

2010 Mathematics Subject Classification. 35R09, 35K67, 74S20.

Key words and phrases. option pricing, well-posedness, difference scheme, rate of convergence.

G. M. Coclite is member of the Gruppo Nazionale per l'Analisi Matematica, la Probabilità e le loro Applicazioni (GNAMPA) of the Istituto Nazionale di Alta Matematica (INdAM). This project has received funding from the European Union's Horizon 2020 research and innovation programme under the Marie Skłodowska-Curie grant agreement No 642768 . 
Option pricing in the Heston model leads to the solution of the bivariate degenerate parabolic PDE

$$
\left\{\begin{array}{l}
\partial_{t} u-\frac{1}{2} \operatorname{tr}\left(\mathcal{Q}(x) D^{2} u\right)+b(x)^{\top} \nabla u(x)=0, \\
u(0, \cdot)=u_{0}
\end{array}\right.
$$

Pricing equations for local-stochastic volatility models are the following fully-non linear PDEs

$$
\left\{\begin{array}{l}
\partial_{t} u-\partial_{x}\left(c_{1}+\sigma(t, x, u) u\right)-\partial_{x}^{2}(\sigma(t, x, u) u)-\partial_{y}\left(c_{2}(y) u\right)-\partial_{y}^{2}\left(c_{3}(y) u\right)=0, \\
u(0, \cdot)=u_{0}
\end{array}\right.
$$

where $c_{2}$ and $c_{3}$ are sufficiently regular. The function $\sigma$ depends on the solution $u$ and takes the following form

$$
\sigma(t, x, u)=c_{4}(t, x) \frac{\int_{\mathbb{R}} u(t, z) d z}{\int_{\mathbb{R}} c_{5}(z) u(t, z) d z},
$$

for some regular function $c_{4}$ and $c_{5}$. Some results on the stochastic analysis for the corresponding SDEs are given in [10]. A particle method for the approximation of solutions of the arising pricing SDEs was provided by [4]. An existence result for the arising PDE under certain assumptions on the coefficients is given in [1]. Discretization schemes have been suggested, but to our best knowledge, a thorough numerical analysis of the corresponding class of differential equations is not available. The aim of this paper is to provide some steps in this direction. We focus on the following class of non-linear PDEs, which are closely related to the pricing equation in local stochastic volatility model

$$
\partial_{t} u=\Delta_{x}(\mathcal{I}[u] u)+\Delta_{y} u, \quad t>0, x \in \mathbb{R}^{M}, y \in \mathbb{R}^{N},
$$

where the operator $\mathcal{I}[\cdot]$ is defined as follows

$$
\mathcal{I}[u](x)=\int_{\mathbb{R}^{N}} b(x, z) u(x, z) d z, \quad x \in \mathbb{R}^{M},
$$

for some suitable function $b$. The fact that both $b$ and $u$ are evaluated in the same $x$ adds several difficulties to the problem. To avoid some of these issues, in this paper we shall approximate the operator $\mathcal{I}[\cdot]$ by

$$
\mathcal{I}_{\delta}[u](x)=\frac{1}{\delta^{M}} \int_{\mathbb{R}^{N}} \int_{B_{\delta}(x)} b(x, z) u(\xi, z) d \xi d z, \quad x \in \mathbb{R}^{M} .
$$

Unfortunately, several of our estimates blow up as $\delta \rightarrow 0$ so we did not obtain any results for (1.6). We shall assume that

$$
\begin{aligned}
\text { (H.1) } b \in C^{4}\left(\mathbb{R}^{M} \times \mathbb{R}^{M} \times \mathbb{R}^{N}\right) & \cap W^{4, \infty}\left(\mathbb{R}^{M} \times \mathbb{R}^{M} \times \mathbb{R}^{N}\right), b \geq 0, \text { and } \\
& \sup _{x \in \mathbb{R}^{M}}\left\|\nabla_{x} b(x, \cdot, \cdot)\right\|_{L^{2}\left(\mathbb{R}^{M} \times \mathbb{R}^{N}\right)} \leq C, \\
& \sup _{x \in \mathbb{R}^{M}}\left\|\Delta_{x} b(x, \cdot, \cdot)\right\|_{L^{2}\left(\mathbb{R}^{M} \times \mathbb{R}^{N}\right)} \leq C, \\
& \sup _{x \in \mathbb{R}^{M}}\left\|b(x, \cdot, \cdot)-b_{0}\right\|_{L^{2}\left(\mathbb{R}^{M} \times \mathbb{R}^{N}\right)} \leq C,
\end{aligned}
$$

for some constants $C>0, b_{0} \in \mathbb{R}$

(H.2) $u_{0} \in H^{1}\left(\mathbb{R}^{M} \times \mathbb{R}^{N}\right) \cap L^{1}\left(\mathbb{R}^{M} \times \mathbb{R}^{N}\right), 0 \leq u_{0} \leq u^{*}$ for some positive constant $u^{*}$.

In all the financial applications we have

$$
\left\|u_{0}\right\|_{L^{1}\left(\mathbb{R}^{M} \times \mathbb{R}^{N}\right)}=1 .
$$

An example of coefficient $b$ satisfying $(\mathbf{H} . \mathbf{1})$ is

$$
b(x, \xi)=\frac{1}{\delta \sqrt{\pi}} e^{-|x-\xi|^{2} / \delta^{2}},
$$

for some $\delta>0$. We use this in our numerical example, see Section 4 . 
Remark 1.1. Our model is a simplification of a more complicated model. We can have several different definitions of the functional $\mathcal{I}[\cdot]$. Two cases deserve to be noted

$$
\begin{aligned}
& \mathcal{I}_{1}[u](x)=\left(\int_{\mathbb{R}^{M}} \int_{\mathbb{R}^{N}} b_{1}(x, \xi, z) u(\xi, z) d \xi d z\right)^{2}, \quad x \in \mathbb{R}^{M}, \\
& \mathcal{I}_{2}[u](x)=\frac{\int_{\mathbb{R}^{M}} \int_{\mathbb{R}^{N}} b_{2}(x, \xi, z) u(\xi, z) d \xi d z}{\int_{\mathbb{R}^{M}} \int_{\mathbb{R}^{N}} b_{3}(x, \xi, z) u(\xi, z) d \xi d z}, \quad x \in \mathbb{R}^{M} .
\end{aligned}
$$

Our arguments can be applied also in these two cases. To do this we do not need to assume that $b_{1} \geq 0$, but we do need to assume that $b_{2} \geq 0$ and that $b_{3} \geq b_{*}>0$.

The rest of this paper is organized as follows. In Section 2 we give the definition of solution to (1.1) and prove that it is well-posed. In Section 3 we introduce a difference numerical scheme for (1.1) and prove its convergence to the solutions of (1.1). We provide also an estimate on the rate of convergence. Finally, in Section 4 we present some numerical experiments based on the scheme introduced in Section 3.

\section{WELL-POSEDNESS}

We use the following definition of solution to (1.1).

Definition 2.1. A function $u:[0, \infty) \times \mathbb{R}^{M} \times \mathbb{R}^{N} \rightarrow \mathbb{R}$ is a solution of (1.1) if

(D.1) $u \in C\left([0, \infty) ; L^{2}\left(\mathbb{R}^{M} \times \mathbb{R}^{N}\right)\right) \cap L^{\infty}\left(0, T ; H^{1}\left(\mathbb{R}^{M} \times \mathbb{R}^{N}\right)\right) \cap H^{1}\left((0, T) \times \mathbb{R}^{M} \times \mathbb{R}^{N}\right)$ for every $T>0$

(D.2) for every test function $\varphi \in C^{\infty}\left(\mathbb{R} \times \mathbb{R}^{M} \times \mathbb{R}^{N}\right)$ with compact support

$$
\begin{aligned}
\int_{0}^{\infty} \int_{\mathbb{R}^{M}} \int_{\mathbb{R}^{N}}\left(u \partial_{t} \varphi-\nabla_{x}(\mathcal{I}[u] u) \cdot \nabla_{x} \varphi-\nabla_{y} u \cdot \nabla_{y} \varphi\right) d t d x d y \\
\quad+\int_{\mathbb{R}^{M}} \int_{\mathbb{R}^{N}} u_{0}(x, y) \varphi(0, x, y) d x d y=0 .
\end{aligned}
$$

We prove the well-posedness of (1.1). The main result of this section is the following.

Theorem 2.1. Assume that (H.1) and (H.2) hold. The Cauchy problem (1.1) admits a solution $u$ in the sense of Definition 2.1 such that

$(2.1) u(t, x, y) \geq 0, \quad\|u(t, \cdot, \cdot)\|_{L^{1}\left(\mathbb{R}^{M} \times \mathbb{R}^{N}\right)}=\left\|u_{0}\right\|_{L^{1}\left(\mathbb{R}^{M} \times \mathbb{R}^{N}\right)}, \quad(t, x, y) \in[0, \infty) \times \mathbb{R}^{M} \times \mathbb{R}^{N}$.

Moreover, if we assume also

$$
b \in C^{6}\left(\mathbb{R}^{M} \times \mathbb{R}^{M} \times \mathbb{R}^{N}\right) \cap W^{6, \infty}\left(\mathbb{R}^{M} \times \mathbb{R}^{M} \times \mathbb{R}^{N}\right), \quad u_{0} \in H^{2}\left(\mathbb{R}^{M} \times \mathbb{R}^{N}\right),
$$

we have the additional regularity on $u$

$$
u \in L^{\infty}\left(0, T ; H^{2}\left(\mathbb{R}^{M} \times \mathbb{R}^{N}\right)\right), \quad T>0 .
$$

Finally, let $u$ and $v$ be two solutions of (1.1) with initial data $u_{0}$ and $v_{0}$ respectively, and assume that (2.2) holds and that $\left\|u_{0}\right\|_{L^{1}\left(\mathbb{R}^{M} \times \mathbb{R}^{N}\right)}=\left\|v_{0}\right\|_{L^{1}\left(\mathbb{R}^{M} \times \mathbb{R}^{N}\right)}$. Then

$$
\|u(t, \cdot, \cdot)-v(t, \cdot, \cdot)\|_{L^{2}\left(\mathbb{R}^{M} \times \mathbb{R}^{N}\right)}^{2} \leq e^{e^{\kappa t}}\left\|u_{0}(t, \cdot, \cdot)-v_{0}(t, \cdot, \cdot)\right\|_{L^{2}\left(\mathbb{R}^{M} \times \mathbb{R}^{N}\right)}^{2},
$$

for every $t \geq 0$ and some constant $\kappa>0$ depending only on $b,\left\|u_{0}\right\|_{L^{1}\left(\mathbb{R}^{M} \times \mathbb{R}^{N}\right)}$, and $\left\|v_{0}\right\|_{L^{1}\left(\mathbb{R}^{M} \times \mathbb{R}^{N}\right)}$.

Our arguments are based on two steps. First we fix $\varepsilon>0$ and study the well-posedness of the approximated problem

$$
\begin{cases}\partial_{t} u_{\varepsilon}=\Delta_{x}\left(\mathcal{I}_{\varepsilon}\left[u_{\varepsilon}\right] u_{\varepsilon}\right)+\Delta_{y} u_{\varepsilon}, & t>0, x \in \mathbb{R}^{N}, y \in \mathbb{R}^{M}, \\ u_{\varepsilon}(0, x, y)=u_{0}(x, y), & x \in \mathbb{R}^{N}, y \in \mathbb{R}^{M}\end{cases}
$$

using a fix point argument, where

$$
\mathcal{I}_{\varepsilon}[u](x)=\int_{\mathbb{R}^{M}} \int_{\mathbb{R}^{N}}(b(x, \xi, z)+\varepsilon) u(\xi, z) d \xi d z+\varepsilon, \quad x \in \mathbb{R}^{M} .
$$

Secondly, we send $\varepsilon \rightarrow 0$ and prove Theorem 2.1 .

We prove the well-posedness of (2.5) for every given $0<\varepsilon<1$. 
Theorem 2.2. Assume that (H.1) and (H.2) hold. The Cauchy problem (1.1) admits a solution $u_{\varepsilon}$ such that (D.1) hold,

(2.6) $u_{\varepsilon}(t, x, y) \geq 0, \quad\left\|u_{\varepsilon}(t, \cdot, \cdot)\right\|_{L^{1}\left(\mathbb{R}^{M} \times \mathbb{R}^{N}\right)}=\left\|u_{0}\right\|_{L^{1}\left(\mathbb{R}^{M} \times \mathbb{R}^{N}\right)}, \quad(t, x, y) \in[0, \infty) \times \mathbb{R}^{M} \times \mathbb{R}^{N}$, and for every test function $\varphi \in C^{\infty}\left(\mathbb{R}^{M} \times \mathbb{R}^{N}\right)$ with compact support

$$
\begin{aligned}
\int_{0}^{\infty} \int_{\mathbb{R}^{M}} \int_{\mathbb{R}^{N}}\left(u_{\varepsilon} \partial_{t} \varphi-\nabla_{x}\left(\mathcal{I}_{\varepsilon}\left[u_{\varepsilon}\right]\right.\right. & \left.\left.u_{\varepsilon}\right) \cdot \nabla_{x} \varphi-\nabla_{y} u_{\varepsilon} \cdot \nabla_{y} \varphi\right) d t d x d y \\
& +\int_{\mathbb{R}^{M}} \int_{\mathbb{R}^{N}} u_{0}(x, y) \varphi(0, x, y) d x d y=0 .
\end{aligned}
$$

Moreover, if we assume also (2.2) we have the additional regularity on $u_{\varepsilon}$

$$
u_{\varepsilon} \in L^{\infty}\left(0, T ; H^{2}\left(\mathbb{R}^{M} \times \mathbb{R}^{N}\right)\right), \quad T>0 .
$$

Finally, if $u_{\varepsilon}$ and $v_{\varepsilon}$ are two solutions of (1.1) with initial data $u_{0}$ and $v_{0}$ respectively such that (2.2) $\left\|u_{0}\right\|_{L^{1}\left(\mathbb{R}^{M} \times \mathbb{R}^{N}\right)}=\left\|v_{0}\right\|_{L^{1}\left(\mathbb{R}^{M} \times \mathbb{R}^{N}\right)}$, then

$$
\left\|u_{\varepsilon}(t, \cdot, \cdot)-v_{\varepsilon}(t, \cdot, \cdot)\right\|_{L^{2}\left(\mathbb{R}^{M} \times \mathbb{R}^{N}\right)}^{2} \leq e^{e^{\kappa t}}\left\|u_{0}(t, \cdot, \cdot)-v_{0}(t, \cdot, \cdot)\right\|_{L^{2}\left(\mathbb{R}^{M} \times \mathbb{R}^{N}\right)}^{2},
$$

for every $t \geq 0$ and some constant $\kappa>0$ depending only on $b,\left\|u_{0}\right\|_{L^{1}\left(\mathbb{R}^{M} \times \mathbb{R}^{N}\right)}$, and $\left\|v_{0}\right\|_{L^{1}\left(\mathbb{R}^{M} \times \mathbb{R}^{N}\right)}$.

Consider the set $\mathcal{B}$ of functions $u:[0, \infty) \times \mathbb{R}^{M} \times \mathbb{R}^{N} \rightarrow \mathbb{R}$ such that

$$
\begin{aligned}
& 0 \leq u(t, x, y) \leq u^{*} e^{\kappa_{0} t}, t \geq 0,(x, y) \in \mathbb{R}^{M} \times \mathbb{R}^{N}, \\
& \|u(t, \cdot, \cdot)\|_{L^{1}\left(\mathbb{R}^{M} \times \mathbb{R}^{N}\right)}=\left\|u_{0}\right\|_{L^{1}\left(\mathbb{R}^{M} \times \mathbb{R}^{N}\right)}, t \geq 0, \\
& u \in H^{1}\left((0, T) \times \mathbb{R}^{M} \times \mathbb{R}^{N}\right), \quad T \geq 0,
\end{aligned}
$$

where

$$
\kappa_{0}=\left\|\Delta_{x} b\right\|_{L^{\infty}\left(\mathbb{R}^{M} \times \mathbb{R}^{M} \times \mathbb{R}^{N}\right)}\left\|u_{0}\right\|_{L^{1}\left(\mathbb{R}^{M} \times \mathbb{R}^{N}\right)} .
$$

Since for every $u \in \mathcal{B}$ we have that

$$
\begin{aligned}
0<\varepsilon & \leq \mathcal{I}_{\varepsilon}[u](t, x)=\int_{\mathbb{R}^{M}} \int_{\mathbb{R}^{N}}(b(x, \xi, z)+\varepsilon) u(\xi, z) d \xi d z+\varepsilon \\
& \leq\left(\|b\|_{L^{\infty}\left(\mathbb{R}^{M} \times \mathbb{R}^{M} \times \mathbb{R}^{N}\right)}+\varepsilon\right)\left\|u_{0}\right\|_{L^{1}\left(\mathbb{R}^{M} \times \mathbb{R}^{N}\right)}+\varepsilon
\end{aligned}
$$

we can define the operator $\mathcal{T}$ such that, for every $u \in \mathcal{B}, v=\mathcal{T}[u]$ is the solution of

$$
\begin{cases}\partial_{t} v=\Delta_{x}\left(\mathcal{I}_{\varepsilon}[u] v\right)+\Delta_{y} v, & t>0, x \in \mathbb{R}^{N}, y \in \mathbb{R}^{M}, \\ v(0, x, y)=u_{0}(x, y), & x \in \mathbb{R}^{N}, y \in \mathbb{R}^{M} .\end{cases}
$$

Lemma 2.1 (Nonnegativity). Let $u \in \mathcal{B}$, we have that

$$
\mathcal{T}[u] \geq 0
$$

Proof. Since

$$
\partial_{t} v-\Delta_{x}\left(\mathcal{I}_{\varepsilon}[u] v\right)-\left.\Delta_{y} v\right|_{v=0}=0, \quad u_{0} \geq 0
$$

0 is a subsolution of (2.11). Therefore, (2.10) and the maximum principle for parabolic equations give (2.12).

Lemma 2.2 ( $L^{1}$ estimate). For any $u \in \mathcal{B}$, we have

$$
\|\mathcal{T}[u](t, \cdot, \cdot)\|_{L^{1}\left(\mathbb{R}^{M} \times \mathbb{R}^{N}\right)}=\left\|u_{0}\right\|_{L^{1}\left(\mathbb{R}^{M} \times \mathbb{R}^{N}\right)},
$$

for every $t \geq 0$.

Proof. Let $v=\mathcal{T}[u]$. By (2.12),

$$
\begin{aligned}
\frac{d}{d t} \int_{\mathbb{R}^{M}} \int_{\mathbb{R}^{N}}|v| d x d y & =\frac{d}{d t} \int_{\mathbb{R}^{M}} \int_{\mathbb{R}^{N}} v d x d y=\int_{\mathbb{R}^{M}} \int_{\mathbb{R}^{N}} \partial_{t} v d x d y \\
& =\int_{\mathbb{R}^{M}} \int_{\mathbb{R}^{N}}\left(\Delta_{x}\left(\mathcal{I}_{\varepsilon}[u] v\right)+\Delta_{y} v\right) d x d y=0 .
\end{aligned}
$$

Therefore, integrating over $(0, t)$ we get $(2.13)$. 
Lemma 2.3 ( $L^{\infty}$ estimate). For any $u \in \mathcal{B}$, we have

$$
\mathcal{T}[u](t, x, y) \leq u^{*} e^{\kappa_{0} t},
$$

for every $t \geq 0$ and $(x, y) \in \mathbb{R}^{M} \times \mathbb{R}^{N}$.

Proof. By (H.1), (2.12), and (2.13)

$$
\begin{aligned}
\left|\Delta_{x} \mathcal{I}_{\varepsilon}[u](t, x)\right| & \leq \int_{\mathbb{R}^{M}} \int_{\mathbb{R}^{N}}\left|\Delta_{x} b(x, \xi, z)\right| u(t, \xi, z) d \xi d z \\
& \leq\left\|\Delta_{x} b\right\|_{L^{\infty}\left(\mathbb{R}^{M} \times \mathbb{R}^{M} \times \mathbb{R}^{N}\right)} \int_{\mathbb{R}^{M}} \int_{\mathbb{R}^{N}} u(t, \xi, z) d \xi d z \\
& =\left\|\Delta_{x} b\right\|_{L^{\infty}\left(\mathbb{R}^{M} \times \mathbb{R}^{M} \times \mathbb{R}^{N}\right)}\left\|u_{0}\right\|_{L^{1}\left(\mathbb{R}^{M} \times \mathbb{R}^{N}\right)}=\kappa_{0} .
\end{aligned}
$$

Therefore

$$
\begin{aligned}
\partial_{t} v-\Delta_{x}\left(\mathcal{I}_{\varepsilon}[u] v\right)-\left.\Delta_{y} v\right|_{v=u^{*} e^{\kappa_{0} t}} & =\kappa_{0} u^{*} e^{\kappa_{0} t}-u^{*} e^{\kappa_{0} t} \Delta_{x} \mathcal{I}_{\varepsilon}[u] \\
& =u^{*} e^{\kappa_{0} t}\left(\kappa_{0}-\Delta_{x} \mathcal{I}_{\varepsilon}[u]\right) \geq 0, \\
\left.u^{*} e^{\kappa_{0} t}\right|_{t=0} & =u^{*} \geq u_{0},
\end{aligned}
$$

namely, $u^{*} e^{\kappa_{0} t}$ is a supersolution of (2.11). Thanks to (2.10) and the maximum principle for parabolic equations we have (2.14).

Lemma 2.4 ( $L^{2}$ estimate). Let $u \in \mathcal{B}$, we have that

$$
\|\mathcal{T}[u](t, \cdot, \cdot)\|_{L^{2}\left(\mathbb{R}^{M} \times \mathbb{R}^{N}\right)} \leq e^{\kappa_{0} t / 2}\left\|u_{0}\right\|_{L^{2}\left(\mathbb{R}^{M} \times \mathbb{R}^{N}\right)},
$$

for every $t \geq 0$.

Proof. Let $v=\mathcal{T}[u]$. By (2.10), (2.12), and (2.15), we have

$$
\begin{aligned}
\frac{d}{d t} \int_{\mathbb{R}^{M}} \int_{\mathbb{R}^{N}} \frac{v^{2}}{2} d x d y & =\int_{\mathbb{R}^{M}} \int_{\mathbb{R}^{N}} v \partial_{t} v d x d y \\
& =\int_{\mathbb{R}^{M}} \int_{\mathbb{R}^{N}} v \Delta_{x}\left(\mathcal{I}_{\varepsilon}[u] v\right) d x d y+\int_{\mathbb{R}^{M}} \int_{\mathbb{R}^{N}} v \Delta_{y} v d x d y \\
& =-\int_{\mathbb{R}^{M}} \int_{\mathbb{R}^{N}} \nabla_{x} v \nabla_{x}\left(\mathcal{I}_{\varepsilon}[u] v\right) d x d y-\underbrace{\int_{\mathbb{R}^{M}} \int_{\mathbb{R}^{N}}\left|\nabla_{y} v\right|^{2} d x d y}_{\leq 0} \\
\leq & \underbrace{-\int_{\mathbb{R}^{M}} \int_{\mathbb{R}^{N}} v \nabla_{x} v \cdot \nabla_{x} \mathcal{I}_{\varepsilon}[u] d x d y}_{\mathbb{R}^{M} \int_{\mathbb{R}^{N}}\left|\nabla_{x} v\right|^{2} \mathcal{I}_{\varepsilon}[u] d x d y} \\
\leq \int_{\mathbb{R}^{M}} \int_{\mathbb{R}^{N}} \frac{v^{2}}{2} \Delta_{x} \mathcal{I}_{\varepsilon}[u] d x d y \leq & \kappa_{0} \int_{\mathbb{R}^{M}} \int_{\mathbb{R}^{N}} v^{2} d x d y .
\end{aligned}
$$

Therefore, the Gronwall Lemma gives (2.16).

Lemma $2.5\left(H^{1}\right.$ estimate in $\left.(x, y)\right)$. Let $u \in \mathcal{B}$, we have that

$$
\begin{array}{r}
\left.\left\|\nabla_{x} \mathcal{T}[u](t, \cdot, \cdot)\right\|_{L^{2}\left(\mathbb{R}^{M} \times \mathbb{R}^{N}\right)}^{2}+\left\|\nabla_{y} \mathcal{T}[u](t, \cdot, \cdot)\right\|_{L^{2}\left(\mathbb{R}^{M} \times \mathbb{R}^{N}\right)}^{2}\right) \\
\leq e^{4 \kappa_{0} t}\left(\left\|\nabla_{x} u_{0}\right\|_{L^{2}\left(\mathbb{R}^{M} \times \mathbb{R}^{N}\right)}^{2}+\left\|\nabla_{y} u_{0}\right\|_{L^{2}\left(\mathbb{R}^{M} \times \mathbb{R}^{N}\right)}^{2}\right) \\
+\frac{\kappa_{1}}{3 \kappa_{0}}\left(e^{4 \kappa_{0} t}-e^{\kappa_{0} t}\right)\left\|u_{0}\right\|_{L^{2}\left(\mathbb{R}^{M} \times \mathbb{R}^{N}\right)}^{2},
\end{array}
$$

for every $t \geq 0$, where

$$
\kappa_{1}=\left\|\left(\Delta_{x}\right)^{2} b\right\|_{L^{\infty}\left(\mathbb{R}^{M} \times \mathbb{R}^{M} \times \mathbb{R}^{N}\right)}\left\|u_{0}\right\|_{L^{1}\left(\mathbb{R}^{M} \times \mathbb{R}^{N}\right)} .
$$


Proof. Set $v=\mathcal{T}[u]$. Arguing as in (2.15) we can prove

$$
\left|\left(\Delta_{x}\right)^{2} \mathcal{I}_{\varepsilon}[u](t, x)\right| \leq \kappa_{1} .
$$

Thanks to (2.10), (2.12), (2.15), (2.16), and (2.18) we have

$$
\begin{aligned}
& \frac{d}{d t} \int_{\mathbb{R}^{M}} \int_{\mathbb{R}^{N}} \frac{\left|\nabla_{x} v\right|^{2}+\left|\nabla_{y} v\right|^{2}}{2} d x d y=\int_{\mathbb{R}^{M}} \int_{\mathbb{R}^{N}}\left(\nabla_{x} v \cdot \nabla_{x} \partial_{t} v+\nabla_{y} v \cdot \nabla_{y} \partial_{t} v\right) d x d y \\
& =\int_{\mathbb{R}^{M}} \int_{\mathbb{R}^{N}}\left(\nabla_{x} v \cdot \nabla_{x} \Delta_{x}\left(\mathcal{I}_{\varepsilon}[u] v\right)+\nabla_{x} v \cdot \nabla_{x} \Delta_{y} v\right) d x d y \\
& +\int_{\mathbb{R}^{M}} \int_{\mathbb{R}^{N}}\left(\nabla_{y} v \cdot \nabla_{y} \Delta_{x}\left(\mathcal{I}_{\varepsilon}[u] v\right)+\nabla_{y} v \cdot \nabla_{y} \Delta_{y} v\right) d x d y \\
& =\sum_{i=1}^{M} \int_{\mathbb{R}^{M}} \int_{\mathbb{R}^{N}}\left(\partial_{x_{i}} v \partial_{x_{i}} \Delta_{x}\left(\mathcal{I}_{\varepsilon}[u] v\right)+\partial_{x_{i}} e v \partial_{x_{i}} \Delta_{y} v\right) d x d y \\
& +\sum_{j=1}^{N} \int_{\mathbb{R}^{M}} \int_{\mathbb{R}^{N}}\left(\partial_{y_{j}} v \partial_{y_{j}} \Delta_{x}\left(\mathcal{I}_{\varepsilon}[u] v\right)+\partial_{y_{j}} v \partial_{y_{j}} \Delta_{y} v\right) d x d y \\
& =-\sum_{i=1}^{M} \int_{\mathbb{R}^{M}} \int_{\mathbb{R}^{N}}(\partial_{x_{i}}^{2} v \Delta_{x}\left(\mathcal{I}_{\varepsilon}[u] v\right)+\underbrace{\left|\partial_{x_{i}} \nabla_{y} v\right|^{2}}_{\geq 0}) d x d y \\
& -\sum_{j=1}^{N} \int_{\mathbb{R}^{M}} \int_{\mathbb{R}^{N}} \partial_{y_{j}} \nabla_{x} v \cdot \nabla_{x}\left(\mathcal{I}_{\varepsilon}[u] \partial_{y_{j}} v\right)+\underbrace{\left|\partial_{y_{j}} \nabla_{y} v\right|^{2}}_{>0} d x d y \\
& \leq-\int_{\mathbb{R}^{M}} \int_{\mathbb{R}^{N}} \Delta_{x} v \Delta_{x} \mathcal{I}_{\varepsilon}[u] v+2 \Delta_{x} v \nabla_{x} \mathcal{I}_{\varepsilon}[u] \cdot \nabla_{x} v+\underbrace{\mathcal{I}_{\varepsilon}[u]\left(\Delta_{x} v\right)^{2}}_{\geq 0} d x d y \\
& \left.-\sum_{j=1}^{N} \int_{\mathbb{R}^{M}} \int_{\mathbb{R}^{N}}(\underbrace{\left|\partial_{y_{j}} \nabla_{x} v\right|^{2} \mathcal{I}_{\varepsilon}[u}_{\geq 0}]+\partial_{y_{j}} \nabla_{x} v \cdot \nabla_{x} \mathcal{I}_{\varepsilon}[u] \partial_{y_{j}} v\right) d x d y \\
& \leq-\int_{\mathbb{R}^{M}} \int_{\mathbb{R}^{N}} \frac{1}{2} \Delta_{x}\left(v^{2}\right) \Delta_{x} \mathcal{I}_{\varepsilon}[u]-\Delta_{x} \mathcal{I}_{\varepsilon}[u]\left(2\left|\nabla_{x} v\right|^{2}+\frac{\left|\nabla_{y} v\right|^{2}}{2}\right) d x d y \\
& =\int_{\mathbb{R}^{M}} \int_{\mathbb{R}^{N}} \frac{1}{2}\left(v^{2}\right)\left(\Delta_{x}\right)^{2} \mathcal{I}_{\varepsilon}[u]+\Delta_{x} \mathcal{I}_{\varepsilon}[u]\left(2\left|\nabla_{x} v\right|^{2}+\frac{\left|\nabla_{y} v\right|^{2}}{2}\right) d x d y \\
& \leq \int_{\mathbb{R}^{M}} \int_{\mathbb{R}^{N}} \frac{\kappa_{1}}{2}\left(v^{2}\right)+2 \kappa_{0}\left(\left|\nabla_{x} v\right|^{2}+\left|\nabla_{y} v\right|^{2}\right) d x d y \\
& \leq \frac{\kappa_{1}}{2} e^{\kappa_{0} t}\left\|u_{0}\right\|_{L^{2}\left(\mathbb{R}^{M} \times \mathbb{R}^{N}\right)}^{2}+2 \kappa_{0} \int_{\mathbb{R}^{M}} \int_{\mathbb{R}^{N}}\left(\left|\nabla_{x} v\right|^{2}+\left|\nabla_{y} v\right|^{2}\right) d x d y .
\end{aligned}
$$

The Gronwall Lemma gives (2.17).

Lemma 2.6 $\left(H^{1}\right.$ estimate in $\left.t\right)$. For $u \in \mathcal{B}$, we have

$$
\left\|\partial_{t} \mathcal{T}[u]\right\|_{L^{2}((0, T) \times \mathbb{R})} \leq \kappa_{2}\left(\left\|\partial_{t} u\right\|_{L^{2}((0, T) \times \mathbb{R})}+\left\|u_{0}\right\|_{H^{1}\left(\mathbb{R}^{M} \times \mathbb{R}^{N}\right)}^{2},\right),
$$

for every $T \geq 0$, where $\kappa_{2}$ is a positive constant depending only on $T,\left\|u_{0}\right\|_{L^{1}\left(\mathbb{R}^{M} \times \mathbb{R}^{N}\right)}$, and $b$.

Proof. Let $v=\mathcal{T}[u]$. Multiplying (2.11) by $\partial_{t} v$ and using (2.10), (2.15), (2.16), and (2.17) we get

$$
\begin{aligned}
\int_{0}^{T} & \int_{\mathbb{R}^{M}} \int_{\mathbb{R}^{N}}\left(\partial_{t} v\right)^{2} d t d x d y \\
& =\int_{0}^{T} \int_{\mathbb{R}^{M}} \int_{\mathbb{R}^{N}} \Delta_{x}\left(\mathcal{I}_{\varepsilon}[u] v\right) \partial_{t} v+\Delta_{y} v \partial_{t} v d t d x d y \\
& =-\int_{0}^{T} \int_{\mathbb{R}^{M}} \int_{\mathbb{R}^{N}} \nabla_{x}\left(\mathcal{I}_{\varepsilon}[u] v\right) \cdot \nabla_{x} \partial_{t} v+\nabla_{y} v \cdot \nabla_{y} \partial_{t} v d t d x d y
\end{aligned}
$$




$$
\begin{aligned}
= & -\int_{0}^{T} \int_{\mathbb{R}^{M}} \int_{\mathbb{R}^{N}} v \nabla_{x} \mathcal{I}_{\varepsilon}[u] \cdot \nabla_{x} \partial_{t} v+\mathcal{I}_{\varepsilon}[u] \nabla_{x} v \cdot \nabla_{x} \partial_{t} v+\nabla_{y} v \cdot \nabla_{y} \partial_{t} v d t d x d y \\
= & \int_{0}^{T} \int_{\mathbb{R}^{M}} \int_{\mathbb{R}^{N}} \Delta_{x} \mathcal{I}_{\varepsilon}[u] v \partial_{t} v+\nabla_{x} v \cdot \nabla_{x} \mathcal{I}_{\varepsilon}[u] \partial_{t} v-\mathcal{I}_{\varepsilon}[u] \partial_{t}\left(\frac{\left|\nabla_{x} v\right|^{2}}{2}\right)-\partial_{t}\left(\frac{\left|\nabla_{y} v\right|^{2}}{2}\right) t d x d y \\
= & \int_{0}^{T} \int_{\mathbb{R}^{M}} \int_{\mathbb{R}^{N}} \Delta_{x} \mathcal{I}_{\varepsilon}[u] v \partial_{t} v+\nabla_{x} v \cdot \nabla_{x} \mathcal{I}_{\varepsilon}[u] \partial_{t} v-\mathcal{I}_{\varepsilon}\left[\partial_{t} u\right] \frac{\left|\nabla_{x} v\right|^{2}}{2} d t d x d y \\
& -\int_{\mathbb{R}^{M}} \int_{\mathbb{R}^{N}} \mathcal{I}_{\varepsilon}[u](T, x, y) \frac{\left|\nabla_{x} v(T, x, y)\right|^{2}}{2}+\frac{\left|\nabla_{y} v(T, x, y)\right|^{2}}{2} d t d x d y \\
& +\int_{\mathbb{R}^{M}} \int_{\mathbb{R}^{N}} \mathcal{I}_{\varepsilon}\left[u_{0}\right] \frac{\left|\nabla_{x} u_{0}\right|^{2}}{2}+\frac{\left|\nabla_{y} u_{0}\right|^{2}}{2} d t d x d y \\
\leq & \int_{0}^{T} \int_{\mathbb{R}^{M}} \int_{\mathbb{R}^{N}} \frac{\left(\partial_{t} v\right)^{2}}{2}+\left(\Delta_{x} \mathcal{I}_{\varepsilon}[u] v\right)^{2}+\left(\nabla_{x} v \cdot \nabla_{x} \mathcal{I}_{\varepsilon}[u]\right)^{2} d t d x d y \\
& +\frac{1}{2} \int_{0}^{T}\left\|\mathcal{I}_{\varepsilon}\left[\partial_{t} u\right](t, \cdot, \cdot)\right\|_{L^{\infty}\left(\mathbb{R}^{M} \times \mathbb{R}^{N}\right)}^{2} d t+\frac{1}{2} \int_{0}^{T} \int_{\mathbb{R}^{M}} \int_{\mathbb{R}^{N}}\left(\frac{\left|\nabla_{x} v\right|^{2}}{2} d x d y\right)^{2} d t d x d y \\
& -\int_{\mathbb{R}^{M}} \int_{\mathbb{R}^{N}} \mathcal{I}_{\varepsilon}[u](T, x, y) \frac{\left|\nabla_{x} v(T, x, y)\right|^{2}}{2}+\frac{\left|\nabla_{y} v(T, x, y)\right|^{2}}{2} d t d x d y \\
& +\int_{\mathbb{R}^{M}} \int_{\mathbb{R}^{N}} \mathcal{I}_{\varepsilon}\left[u_{0}\right] \frac{\left|\nabla_{x} u_{0}\right|^{2}}{2}+\frac{\left|\nabla_{y} u_{0}\right|^{2}}{2} d t d x d y \\
\leq & c\left\|u_{0}\right\|_{H^{1}\left(\mathbb{R}^{M} \times \mathbb{R}^{N}\right)}^{2}+\int_{0}^{T} \int_{\mathbb{R}^{M}} \int_{\mathbb{R}^{N}} \frac{\left(\partial_{t} v\right)^{2}}{2} d t d x d y+\frac{1}{2} \int_{0}^{T}\left\|\mathcal{I}_{\varepsilon}\left[\partial_{t} u\right](t, \cdot)\right\|_{L^{\infty}\left(\mathbb{R}^{M}\right)}^{2} d t,
\end{aligned}
$$

where $\kappa_{2}$ is a positive constant depending only on $T,\left\|u_{0}\right\|_{L^{1}\left(\mathbb{R}^{M} \times \mathbb{R}^{N}\right)}$, and $b$. Using the Hölder inequality,

$$
\begin{aligned}
\left|\mathcal{I}_{\varepsilon}\left[\partial_{t} u\right](t, x)\right| & =\left|\int_{\mathbb{R}^{M}} \int_{\mathbb{R}^{N}} b(x, \xi, z) \partial_{t} u(t, \xi, z) d \xi d z\right| \\
& =\left|\int_{\mathbb{R}^{M}} \int_{\mathbb{R}^{N}}\left(b(x, \xi, z)-b_{0}\right) \partial_{t} u(t, \xi, z) d \xi d z+b_{0} \frac{d}{d t} \int_{\mathbb{R}} u(t, y) d \xi d z\right| \\
& =\left|\int_{\mathbb{R}^{M}} \int_{\mathbb{R}^{N}}\left(b(x, \xi, z)-b_{0}\right) \partial_{t} u(t, \xi, z) d \xi d z\right| \\
& \leq \int_{\mathbb{R}^{M}} \int_{\mathbb{R}^{N}}\left|b(x, \xi, z)-b_{0}\right|\left|\partial_{t} u(t, \xi, z)\right| d \xi d z \\
& \leq\left\|b(x, \cdot \cdot \cdot)-b_{0}\right\|_{L^{2}\left(\mathbb{R}^{M} \times \mathbb{R}^{N}\right)}\left\|\partial_{t} u(t, \cdot, \cdot)\right\|_{L^{2}\left(\mathbb{R}^{M} \times \mathbb{R}^{N}\right)}
\end{aligned}
$$

we get

$$
\frac{1}{2} \int_{0}^{T} \int_{\mathbb{R}^{M}} \int_{\mathbb{R}^{N}}\left(\partial_{t} v\right)^{2} d t d x d y \leq c\left\|u_{0}\right\|_{H^{1}\left(\mathbb{R}^{M} \times \mathbb{R}^{N}\right)}^{2}+c\left\|\partial_{t} u\right\|_{L^{2}\left((0, T) \times \mathbb{R}^{M} \times \mathbb{R}^{N}\right)}^{2} .
$$

Lemma 2.7 (Continuity). For every $u, \bar{u} \in \mathcal{B}$, we have that

$$
\|\mathcal{T}[u]-\mathcal{T}[\bar{u}]\|_{L^{\infty}\left(0, T ; L^{2}\left(\mathbb{R}^{M} \times \mathbb{R}^{N}\right)\right)} \leq \kappa_{3} T^{1 / 4} e^{\kappa_{3} T} \sqrt{\|u-\bar{u}\|_{\left.L^{\infty}\left((0, T) \times \mathbb{R}^{M} \times \mathbb{R}^{N}\right)\right)}},
$$

for every $T \geq 0$ and some constant $\kappa_{3}>0$ depending only on $b$, $u_{0}$, and $v_{0}$.

Proof. Define

We have that

$$
v=\mathcal{T}[u], \quad \bar{v}=\mathcal{T}[\bar{u}], \quad w=v-\bar{v} .
$$

$$
w(0, x)=0, \quad \partial_{t} w=\Delta_{x}\left(\mathcal{I}_{\varepsilon}[u] w+\left(\mathcal{I}_{\varepsilon}[u]-\mathcal{I}_{\varepsilon}[\bar{u}]\right) \bar{v}\right)+\Delta_{y} w .
$$

We begin with some observations regarding the nonlocal term $\mathcal{I}_{\varepsilon}[u]-\mathcal{I}_{\varepsilon}[\bar{u}]$

$$
\left(\mathcal{I}_{\varepsilon}[u]-\mathcal{I}_{\varepsilon}[\bar{u}]\right)(t, x)=\int_{\mathbb{R}^{M}} \int_{\mathbb{R}^{N}} b(x, \xi, z)(u(t, \xi, z)-\bar{u}(t, \xi, z)) d \xi d z
$$




$$
\begin{aligned}
& +\varepsilon \int_{\mathbb{R}^{M}} \int_{\mathbb{R}^{N}}(u(t, \xi, z)-\bar{u}(t, \xi, z)) d \xi d z \\
= & \int_{\mathbb{R}^{M}} \int_{\mathbb{R}^{N}} b(x, \xi, z)(u(t, \xi, z)-\bar{u}(t, \xi, z)) d \xi d z \\
& +\varepsilon \underbrace{\left(\|u(t, \cdot, \cdot)\|_{L^{1}\left(\mathbb{R}^{M} \times \mathbb{R}^{N}\right)}-\|\bar{u}(t, \cdot, \cdot)\|_{\left.L^{1}\left(\mathbb{R}^{M} \times \mathbb{R}^{N}\right)\right)}\right.}_{=0} \\
= & \int_{\mathbb{R}^{M}} \int_{\mathbb{R}^{N}} b(x, \xi, z)(u(t, \xi, z)-\bar{u}(t, \xi, z)) d \xi d z \\
= & \int_{\mathbb{R}^{M}} \int_{\mathbb{R}^{N}}\left(b(x, \xi, z)-b_{0}\right)(u(t, \xi, z)-\bar{u}(t, \xi, z)) d \xi d z, \\
\nabla_{x}\left(\mathcal{I}_{\varepsilon}[u]-\mathcal{I}_{\varepsilon}[\bar{u}]\right)(t, x)= & \int_{\mathbb{R}^{M}} \int_{\mathbb{R}^{N}} \nabla_{x} b(x, \xi, z)(u(t, \xi, z)-\bar{u}(t, \xi, z)) d \xi d z, \\
\Delta_{x}\left(\mathcal{I}_{\varepsilon}[u]-\mathcal{I}_{\varepsilon}[\bar{u}]\right)(t, x)= & \int_{\mathbb{R}^{M}} \int_{\mathbb{R}^{N}} \Delta_{x} b(x, \xi, z)(u(t, \xi, z)-\bar{u}(t, \xi, z)) d \xi d z .
\end{aligned}
$$

Using the Hölder inequality,

$$
\begin{aligned}
\left|\left(\mathcal{I}_{\varepsilon}[u]-\mathcal{I}_{\varepsilon}[\bar{u}]\right)(t, x)\right| & \leq \int_{\mathbb{R}^{M}} \int_{\mathbb{R}^{N}}\left|b(x, \xi, z)-b_{0} \| u(t, \xi, z)-\bar{u}(t, \xi, z)\right| d \xi d z \\
& \leq\left\|b(x, \cdot, \cdot)-b_{0}\right\|_{L^{2}\left(\mathbb{R}^{M} \times \mathbb{R}^{N}\right)}\|u(t, \cdot, \cdot)-\bar{u}(t, \cdot, \cdot)\|_{L^{2}\left(\mathbb{R}^{M} \times \mathbb{R}^{N}\right)}, \\
\left|\partial_{x}\left(\mathcal{I}_{\varepsilon}[u]-\mathcal{I}_{\varepsilon}[\bar{u}]\right)(t, x)\right| & \leq \int_{\mathbb{R}^{M}} \int_{\mathbb{R}^{N}}\left|\partial_{x} b(x, \xi, z) \| u(t, \xi, z)-\bar{u}(t, \xi, z)\right| d \xi d z \\
& \leq\left\|\partial_{x} b(x, \cdot, \cdot)\right\|_{L^{2}\left(\mathbb{R}^{M} \times \mathbb{R}^{N}\right)}\|u(t, \cdot, \cdot)-\bar{u}(t, \cdot, \cdot)\|_{L^{2}\left(\mathbb{R}^{M} \times \mathbb{R}^{N}\right)}, \\
\left|\partial_{x}^{2}\left(\mathcal{I}_{\varepsilon}[u]-\mathcal{I}_{\varepsilon}[\bar{u}]\right)(t, x)\right| & \leq \int_{\mathbb{R}^{M}} \int_{\mathbb{R}^{N}}\left|\partial_{x}^{2} b(x, \xi, z) \| u(t, \xi, z)-\bar{u}(t, \xi, z)\right| d \xi d z \\
& \leq\left\|\partial_{x}^{2} b(x, \cdot, \cdot)\right\|_{L^{2}\left(\mathbb{R}^{M} \times \mathbb{R}^{N}\right)}\|u(t, \cdot, \cdot)-\bar{u}(t, \cdot, \cdot)\|_{L^{2}\left(\mathbb{R}^{M} \times \mathbb{R}^{N}\right)} .
\end{aligned}
$$

Therefore we have

$$
\begin{array}{r}
\left|\left(\mathcal{I}_{\varepsilon}[u]-\mathcal{I}_{\varepsilon}[\bar{u}]\right)(t, x)\right| \leq C\|u(t, \cdot, \cdot)-\bar{u}(t, \cdot, \cdot)\|_{L^{2}\left(\mathbb{R}^{M} \times \mathbb{R}^{N}\right)}, \\
\left|\partial_{x}\left(\mathcal{I}_{\varepsilon}[u]-\mathcal{I}_{\varepsilon}[\bar{u}]\right)(t, x)\right| \leq C\|u(t, \cdot, \cdot)-\bar{u}(t, \cdot, \cdot)\|_{L^{2}\left(\mathbb{R}^{M} \times \mathbb{R}^{N}\right)}, \\
\left|\partial_{x}^{2}\left(\mathcal{I}_{\varepsilon}[u]-\mathcal{I}_{\varepsilon}[\bar{u}]\right)(t, x)\right| \leq C\|u(t, \cdot, \cdot)-\bar{u}(t, \cdot, \cdot)\|_{L^{2}\left(\mathbb{R}^{M} \times \mathbb{R}^{N}\right)} .
\end{array}
$$

Thanks to the Hölder inequality, (2.10), (2.15), (2.16), (2.17), and (2.21), we have for every $0<t<T$,

$$
\begin{aligned}
\frac{d}{d t} & \int_{\mathbb{R}^{M}} \int_{\mathbb{R}^{N}} \frac{w^{2}}{2} d x d y=\int_{\mathbb{R}^{M}} \int_{\mathbb{R}^{N}} w \partial_{t} w d x d y \\
= & \int_{\mathbb{R}^{M}} \int_{\mathbb{R}^{N}} w \Delta_{x}\left(\mathcal{I}_{\varepsilon}[u] w\right)+w \Delta_{x}\left(\left(\mathcal{I}_{\varepsilon}[u]-\mathcal{I}_{\varepsilon}[\bar{u}]\right) \bar{v}\right)+w \Delta_{y} w d x d y \\
= & -\int_{\mathbb{R}^{M}} \int_{\mathbb{R}^{N}} \nabla_{x} w \cdot \nabla_{x}(\mathcal{I}[u] w)+\nabla_{x} w \cdot \nabla_{x}\left(\left(\mathcal{I}_{\varepsilon}[u]-\mathcal{I}_{\varepsilon}[\bar{u}]\right) \bar{v}\right)+\underbrace{\left|\nabla_{y} w\right|^{2}}_{\geq 0} d x d y \\
\leq & -\int_{\mathbb{R}^{M}} \int_{\mathbb{R}^{N}} \underbrace{\left|\nabla_{x} w\right|^{2} \mathcal{I}_{\varepsilon}[u]}_{\geq 0}+w \nabla_{x} w \cdot \nabla_{x} \mathcal{I}_{\varepsilon}[u] d x d y \\
& \left.+\int_{\mathbb{R}^{M}} \int_{\mathbb{R}^{N}} \nabla_{x} w \cdot \nabla_{x}\left(\mathcal{I}_{\varepsilon}[u]-\mathcal{I}_{\varepsilon}[\bar{u}]\right) \bar{v}+\nabla_{x} w \cdot \nabla_{x} \bar{v}\left(\mathcal{I}_{\varepsilon}[u]-\mathcal{I}_{\varepsilon}[\bar{u}]\right)\right) d x d y \\
\leq & \int_{\mathbb{R}^{M}} \int_{\mathbb{R}^{N}}\left(\frac{w^{2}}{2} \Delta_{x} \mathcal{I}_{\varepsilon}[u]+\nabla_{x} w \cdot \nabla_{x}\left(\mathcal{I}_{\varepsilon}[u]-\mathcal{I}_{\varepsilon}[\bar{u}]\right) \bar{v}+\nabla_{x} w \cdot \nabla_{x} \bar{v}\left(\mathcal{I}_{\varepsilon}[u]-\mathcal{I}_{\varepsilon}[\bar{u}]\right)\right) d x d y \\
\leq & \left\|\Delta_{x} \mathcal{I}_{\varepsilon}[u](t, \cdot, \cdot)\right\|_{L^{\infty}\left(\mathbb{R}^{M} \times \mathbb{R}^{N}\right)} \int_{\mathbb{R}^{M}} \int_{\mathbb{R}^{N}} \frac{w^{2}}{2} d x d y
\end{aligned}
$$




$$
\begin{aligned}
& +\left\|\nabla_{x}\left(\mathcal{I}_{\varepsilon}[u]-\mathcal{I}_{\varepsilon}[\bar{u}]\right)(t, \cdot, \cdot)\right\|_{L^{\infty}\left(\mathbb{R}^{M} \times \mathbb{R}^{N}\right)} \int_{\mathbb{R}^{M}} \int_{\mathbb{R}^{N}}\left(\left|\nabla_{x} v\right|+\left|\nabla_{x} \bar{v}\right|\right) \bar{v} d x d y \\
+ & \left\|\left(\mathcal{I}_{\varepsilon}[u]-\mathcal{I}_{\varepsilon}[\bar{u}]\right)(t, \cdot, \cdot)\right\|_{L^{\infty}\left(\mathbb{R}^{M} \times \mathbb{R}^{N}\right)} \int_{\mathbb{R}^{M}} \int_{\mathbb{R}^{N}}\left(\left|\nabla_{x} v\right|+\left|\nabla_{x} \bar{v}\right|\right)\left|\nabla_{x} \bar{v}\right| d x d y \\
\leq & \frac{\kappa_{3}}{2} \int_{\mathbb{R}^{M}} \int_{\mathbb{R}^{N}} w^{2} d x d y+\frac{\kappa_{3}}{2} e^{\kappa_{3} t}\|u(t, \cdot, \cdot)-\bar{u}(t, \cdot, \cdot)\|_{L^{2}\left(\mathbb{R}^{M} \times \mathbb{R}^{N}\right)},
\end{aligned}
$$

for some constant $\kappa_{3}$ depending on $u_{0}, v_{0}$, and $b$. Thanks to the Gronwall Lemma and (2.21) we have, for every $0<t<T$,

$$
\begin{aligned}
& \|w(t, \cdot, \cdot)\|_{L^{2}\left(\mathbb{R}^{M} \times \mathbb{R}^{N}\right)}^{2} \leq \kappa_{3} e^{\kappa_{3} t} \int_{0}^{t}\|u(s, \cdot, \cdot)-\bar{u}(s, \cdot, \cdot)\|_{L^{2}\left(\mathbb{R}^{M} \times \mathbb{R}^{N}\right)} d s \\
& \leq \kappa_{3} e^{\kappa_{3} t} \sqrt{t} \sqrt{\int_{0}^{t}\|u(s, \cdot, \cdot)-\bar{u}(s, \cdot, \cdot)\|_{L^{2}\left(\mathbb{R}^{M} \times \mathbb{R}^{N}\right)}^{2} d s}
\end{aligned}
$$

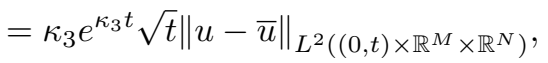

that gives (2.20) when $t=T$.

Lemma 2.8 (Invariance). We have that

$$
\mathcal{T}[\mathcal{B}] \subset \mathcal{B} .
$$

Proof. This is a direct consequence of Lemmas 2.1, 2.2, 2.3, 2.4, 2.5, and 2.6.

Lemma 2.9 (Compactness). We have that

$$
\mathcal{T}[\mathcal{B}] \subset \subset \mathcal{B} .
$$

Proof. Let $\left\{u_{n}\right\}_{n} \subset \mathcal{B}$ be a bounded sequence and $T>0$ be given. Lemmas 2.4, 2.5, and 2.6 guarantee that

$$
\begin{aligned}
& \left\{u_{n}\right\}_{n} \text { is bounded in } L^{\infty}\left(0, T ; H^{1}\left(\mathbb{R}^{M} \times \mathbb{R}^{N}\right)\right), \\
& \left\{\partial_{t} u_{n}\right\}_{n} \text { is bounded in } L^{2}\left((0, T) \times \mathbb{R}^{M} \times \mathbb{R}^{N}\right) .
\end{aligned}
$$

Since (see [8])

$$
H^{1}\left(\mathbb{R}^{M} \times \mathbb{R}^{N}\right) \subset \subset L_{\mathrm{loc}}^{2}\left(\mathbb{R}^{M} \times \mathbb{R}^{N}\right),
$$

using [9, Theorem 5], there exists a subsequence $\left\{u_{n_{k}}\right\}_{k}$ and a function $u$ such that

$$
u_{n_{k}} \rightarrow u \quad \text { in } L^{p}\left(0, T ; L^{2}(\mathbb{R})\right), 1 \leq p<\infty .
$$

Lemma 2.10 (Existence). The Cauchy problem (1.1) admits a solution $u_{\varepsilon}$ such that (D.1), (2.1), and (2.7) hold.

Proof. The existence of a solution for (2.5) follows from the previous lemmas and the Schauder fixed point theorem.

Lemma 2.11 (Additional Regularity). Let $u_{\varepsilon}$ solve (2.5). If (2.2) holds we have that

$$
\begin{aligned}
\left\|D_{x, y}^{2} \mathcal{T}[u](t, \cdot)\right\|_{L^{2}\left(\mathbb{R}^{M} \times \mathbb{R}^{N}\right)} & \leq e^{\kappa_{4} t}\left\|D_{x, y}^{2} u_{0}\right\|_{L^{2}\left(\mathbb{R}^{M} \times \mathbb{R}^{N}\right)}+\kappa_{4} e^{\kappa_{4} t} t\left\|u_{0}\right\|_{H^{1}\left(\mathbb{R}^{M} \times \mathbb{R}^{N}\right)}, \\
\left\|\mathcal{I}_{\varepsilon}[u] D_{x, y}^{3} \mathcal{T}[u]^{2}\right\|_{L^{1}\left((0, t) \times \mathbb{R}^{M} \times \mathbb{R}^{N}\right)}^{2} & \leq e^{\kappa_{4} t}\left\|D_{x, y}^{2} u_{0}\right\|_{L^{2}\left(\mathbb{R}^{M} \times \mathbb{R}^{N}\right)}^{2}+\kappa_{4} e^{\kappa_{4} t} t\left\|u_{0}\right\|_{H^{1}\left(\mathbb{R}^{M} \times \mathbb{R}^{N}\right)}^{2},
\end{aligned}
$$

for every $t \geq 0$, where $\kappa_{4}>0$ depends on $b$ and $\left\|u_{0}\right\|_{L^{1}\left(\mathbb{R}^{M} \times \mathbb{R}^{N}\right)}$.

Proof. Set $v=\mathcal{T}[u]$. Thanks to (2.10), (2.15), (2.16), (2.17), and (2.19), we have

$$
\begin{aligned}
& \frac{d}{d t} \int_{\mathbb{R}^{M}} \int_{\mathbb{R}^{N}} \frac{\left|D_{x, y}^{2} v\right|^{2}}{2} d x d y=\frac{d}{d t} \sum_{i, j=1}^{M+N} \int_{\mathbb{R}^{M}} \int_{\mathbb{R}^{N}} \frac{\left|\partial_{i j}^{2} v\right|^{2}}{2} d x d y \\
& \quad=\sum_{i, j=1}^{M+N} \int_{\mathbb{R}^{M}} \int_{\mathbb{R}^{N}} \partial_{i j}^{2} v \partial_{i j}^{2} \partial_{t} v d x d y=
\end{aligned}
$$




$$
\begin{aligned}
& =\sum_{i, j=1}^{M+N} \int_{\mathbb{R}^{M}} \int_{\mathbb{R}^{N}} \partial_{i j}^{2} v \Delta_{x} \partial_{i j}^{2}\left(\mathcal{I}_{\varepsilon}[u] v\right)+\partial_{i j}^{2} v \Delta_{y} \partial_{i j}^{2} v v d x d y \\
& =-\sum_{i, j=1}^{M+N} \int_{\mathbb{R}^{M}} \int_{\mathbb{R}^{N}} \nabla_{x} \partial_{i j}^{2} v \cdot \nabla_{x} \partial_{i j}^{2}\left(\mathcal{I}_{\varepsilon}[u] v\right)+\underbrace{\left|\nabla_{y} \partial_{i j}^{2} v\right|^{2}}_{\geq 0} d x d y \\
& \leq-\sum_{i, j=1}^{M+N} \int_{\mathbb{R}^{M}} \int_{\mathbb{R}^{N}} \nabla_{x} \partial_{i j}^{2} v \cdot \nabla_{x} \partial_{i j}^{2} \mathcal{I}_{\varepsilon}[u] v+\nabla_{x} \partial_{i j}^{2} v \cdot \nabla_{x} v \partial_{i j}^{2} \mathcal{I}_{\varepsilon}[u] d x d y \\
& -2 \sum_{i, j=1}^{M+N} \int_{\mathbb{R}^{M}} \int_{\mathbb{R}^{N}} \nabla_{x} \partial_{i j}^{2} v \cdot \nabla_{x} \partial_{i} \mathcal{I}_{\varepsilon}[u] \partial_{j} v+\nabla_{x} \partial_{i j}^{2} v \cdot \nabla_{x} \partial_{i} v \partial_{j} \mathcal{I}_{\varepsilon}[u] d x d y \\
& -\sum_{i, j=1}^{M+N} \int_{\mathbb{R}^{M}} \int_{\mathbb{R}^{N}} \nabla_{x} \partial_{i j}^{2} v \cdot \nabla_{x} \mathcal{I}_{\varepsilon}[u] \partial_{i j}^{2} v+\left|\nabla_{y} \partial_{i j}^{2} v\right|^{2}+\underbrace{\left|\nabla_{x} \partial_{i j}^{2} v\right|^{2} \mathcal{I}_{\varepsilon}[u]}_{\geq 0} d x d y \\
& \leq-\sum_{i, j=1}^{M+N} \int_{\mathbb{R}^{M}} \int_{\mathbb{R}^{N}} \nabla_{x}\left(\frac{\partial_{i j}^{2}\left(v^{2}\right)}{2}\right) \cdot \nabla_{x} \partial_{i j}^{2} \mathcal{I}_{\varepsilon}[u]-\partial_{i}\left(\nabla_{x} v \partial_{j} v\right) \cdot \nabla_{x} \partial_{i j}^{2} \mathcal{I}_{\varepsilon}[u]-\partial_{i} v \nabla_{x} \partial_{j} v \cdot \nabla_{x} \partial_{i j}^{2} \mathcal{I}_{\varepsilon}[u] d x d y \\
& -\sum_{i, j=1}^{M+N} \int_{\mathbb{R}^{M}} \int_{\mathbb{R}^{N}} \frac{\partial_{i j}^{2}\left(\left|\nabla_{x} v\right|^{2}\right)}{2} \partial_{i j}^{2} \mathcal{I}_{\varepsilon}[u]-\nabla_{x} \partial_{i} v \cdot \nabla_{x} \partial_{j} v \mathcal{I}_{\varepsilon}[u] d x d y \\
& -\sum_{i, j=1}^{M+N} \int_{\mathbb{R}^{M}} \int_{\mathbb{R}^{N}} \nabla_{x} \partial_{i}\left(\left|\partial_{j} v\right|^{2}\right) \cdot \nabla_{x} \partial_{i} \mathcal{I}_{\varepsilon}[u]-2 \partial_{i j}^{2} v \nabla_{x} \partial_{j} v \cdot \nabla_{x} \partial_{i} \mathcal{I}_{\varepsilon}[u]+\partial_{j}\left(\left|\nabla_{x} \partial_{i} v\right|^{2}\right) \partial_{j} \mathcal{I}_{\varepsilon}[u] d x d y \\
& -\frac{1}{2} \sum_{i, j=1}^{M+N} \int_{\mathbb{R}^{M}} \int_{\mathbb{R}^{N}} \nabla_{x}\left(\left|\partial_{i j}^{2} v\right|^{2}\right) \cdot \nabla_{x} \mathcal{I}_{\varepsilon}[u]+\left|\nabla_{y} \partial_{i j}^{2} v\right|^{2}+\left|\nabla_{x} \partial_{i j}^{2} v\right|^{2} \mathcal{I}_{\varepsilon}[u] d x d y \\
& \leq \sum_{i, j=1}^{M+N} \int_{\mathbb{R}^{M}} \int_{\mathbb{R}^{N}} \frac{v^{2}}{2} \Delta_{x} \partial_{i i j j}^{4} \mathcal{I}_{\varepsilon}[u]+\partial_{i}\left(\nabla_{x} v \partial_{j} v\right) \cdot \nabla_{x} \partial_{i j}^{2} \mathcal{I}_{\varepsilon}[u]+\partial_{i} v \nabla_{x} \partial_{j} v \cdot \nabla_{x} \partial_{i j}^{2} \mathcal{I}_{\varepsilon}[u] d x d y \\
& -\sum_{i, j=1}^{M+N} \int_{\mathbb{R}^{M}} \int_{\mathbb{R}^{N}} \frac{\left|\nabla_{x} v\right|^{2}}{2} \partial_{i i j j}^{4} \mathcal{I}_{\varepsilon}[u]-\nabla_{x} \partial_{i} v \cdot \nabla_{x} \partial_{j} v \mathcal{I}_{\varepsilon}[u] d x d y \\
& -\sum_{i, j=1}^{M+N} \int_{\mathbb{R}^{M}} \int_{\mathbb{R}^{N}}\left|\partial_{j} v\right|^{2} \Delta_{x} \partial_{i i}^{2} \mathcal{I}_{\varepsilon}[u]-2 \partial_{i j}^{2} v \nabla_{x} \partial_{j} v \cdot \nabla_{x} \partial_{i} \mathcal{I}_{\varepsilon}[u]-\left|\nabla_{x} \partial_{i} v\right|^{2} \partial_{i j}^{2} \mathcal{I}_{\varepsilon}[u] d x d y \\
& +\frac{1}{2} \sum_{i, j=1}^{M+N} \int_{\mathbb{R}^{M}} \int_{\mathbb{R}^{N}}\left|\partial_{i j}^{2} v\right|^{2} \Delta_{x} \mathcal{I}_{\varepsilon}[u]+\left|\nabla_{y} \partial_{i j}^{2} v\right|^{2}+\left|\nabla_{x} \partial_{i j}^{2} v\right|^{2} \mathcal{I}_{\varepsilon}[u] d x d y \\
& \leq \kappa_{4} \int_{\mathbb{R}^{M}} \int_{\mathbb{R}^{N}} \frac{\left(D_{(x, y)}^{2} v\right)^{2}}{2}+\left|\nabla_{x} v\right|^{2}+\left|\nabla_{y} v\right|^{2}+v^{2}+\left|\nabla_{y} \partial_{i j}^{2} v\right|^{2}+\left|\nabla_{x} \partial_{i j}^{2} v\right|^{2} \mathcal{I}_{\varepsilon}[u] d x d y \\
& \leq \kappa_{4} \int_{\mathbb{R}^{M}} \int_{\mathbb{R}^{N}} \frac{\left(D_{(x, y)}^{2} v\right)^{2}}{2}+\left|\nabla_{y} \partial_{i j}^{2} v\right|^{2}+\left|\nabla_{x} \partial_{i j}^{2} v\right|^{2} \mathcal{I}_{\varepsilon}[u] d x d y+\frac{\kappa_{4}}{2} e^{\kappa_{4} t}\left\|u_{0}\right\|_{H^{1}\left(\mathbb{R}^{M} \times \mathbb{R}^{N}\right)}^{2},
\end{aligned}
$$

where $\kappa_{4}>0$ depends on $b$ and $\left\|u_{0}\right\|_{L^{1}\left(\mathbb{R}^{M} \times \mathbb{R}^{N}\right)}$. The Gronwall Lemma gives (2.26).

Lemma 2.12 (Uniqueness and Stability). Let $u_{\varepsilon}$ and $v_{\varepsilon}$ be two solutions of (1.1) with initial data $u_{0}$ and $v_{0}$ respectively. If $(2.2)$ holds and $\left\|u_{0}\right\|_{L^{1}\left(\mathbb{R}^{M} \times \mathbb{R}^{N}\right)}=\left\|v_{0}\right\|_{L^{1}\left(\mathbb{R}^{M} \times \mathbb{R}^{N}\right)}$, then the stability estimate (2.9) holds.

Proof. The difference

$$
w_{\varepsilon}=u_{\varepsilon}-v_{\varepsilon}
$$


solves the problem

$$
\begin{cases}\partial_{t} w_{\varepsilon}=\Delta_{x}\left(\mathcal{I}\left[u_{\varepsilon}\right] w_{\varepsilon}+\mathcal{I}_{\varepsilon}\left[w_{\varepsilon}\right] v_{\varepsilon}\right)+\Delta_{y} w_{\varepsilon}, & t>0, \quad(x, y) \in \mathbb{R}^{M} \times \mathbb{R}^{N}, \\ w_{\varepsilon}(0, x, y)=u_{0}(x, y)-v_{0}(x, y), & (x, y) \in \mathbb{R}^{M} \times \mathbb{R}^{N} .\end{cases}
$$

Arguing as in (2.22), we can prove

$$
\begin{array}{r}
\left|\left(\mathcal{I}_{\varepsilon}\left[w_{\varepsilon}\right]\right)(t, x)\right| \leq C\left\|w_{\varepsilon}(t, \cdot, \cdot)\right\|_{L^{2}\left(\mathbb{R}^{M} \times \mathbb{R}^{N}\right)}, \\
\left|\partial_{x} \mathcal{I}_{\varepsilon}\left[w_{\varepsilon}\right](t, x)\right| \leq C\left\|w_{\varepsilon}(t, \cdot, \cdot)\right\|_{L^{2}\left(\mathbb{R}^{M} \times \mathbb{R}^{N}\right)}, \\
\left|\partial_{x}^{2} \mathcal{I}_{\varepsilon}\left[w_{\varepsilon}\right](t, x)\right| \leq C\left\|w_{\varepsilon}(t, \cdot, \cdot)\right\|_{L^{2}\left(\mathbb{R}^{M} \times \mathbb{R}^{N}\right)} .
\end{array}
$$

Thanks to the Hölder inequality, (2.10), (2.12), (2.16), (2.17), and (2.26), we have for every $0<t<T$,

$$
\begin{aligned}
\frac{d}{d t} & \int_{\mathbb{R}^{M}} \int_{\mathbb{R}^{N}} \frac{w_{\varepsilon}^{2}}{2} d x d y=\int_{\mathbb{R}^{M}} \int_{\mathbb{R}^{N}} w_{\varepsilon} \partial_{t} w_{\varepsilon} d x d y \\
= & \int_{\mathbb{R}^{M}} \int_{\mathbb{R}^{N}} w_{\varepsilon} \Delta_{x}\left(\mathcal{I}_{\varepsilon}\left[u_{\varepsilon}\right] w_{\varepsilon}\right)+w_{\varepsilon} \Delta_{x}\left(\mathcal{I}_{\varepsilon}\left[w_{\varepsilon}\right] v_{\varepsilon}\right)+w_{\varepsilon} \Delta_{y} w_{\varepsilon} d x d y \\
= & \int_{\mathbb{R}^{M}} \int_{\mathbb{R}^{N}}-\nabla_{x} w_{\varepsilon} \cdot \nabla_{x}\left(\mathcal{I}_{\varepsilon}\left[u_{\varepsilon}\right] w_{\varepsilon}\right)+w_{\varepsilon} \Delta_{x}\left(\mathcal{I}_{\varepsilon}\left[w_{\varepsilon}\right] v_{\varepsilon}\right) \underbrace{-\left|\nabla_{y} w_{\varepsilon}\right|^{2}}_{\leq 0} d x d y \\
\leq & \int_{\mathbb{R}^{M}} \int_{\mathbb{R}^{N}} \underbrace{-\left|\nabla_{x} w_{\varepsilon}\right|^{2} \mathcal{I}_{\varepsilon}\left[u_{\varepsilon}\right]}_{\leq 0}-w_{\varepsilon} \nabla_{x} w_{\varepsilon} \cdot \nabla_{x} \mathcal{I}_{\varepsilon}\left[u_{\varepsilon}\right] d x d y \\
& +\int_{\mathbb{R}^{M}} \int_{\mathbb{R}^{N}} w_{\varepsilon} \Delta_{x} \mathcal{I}_{\varepsilon}\left[w_{\varepsilon}\right] v_{\varepsilon}+2 w_{\varepsilon} \nabla_{x} \mathcal{I}_{\varepsilon}\left[w_{\varepsilon}\right] \cdot \nabla_{x} v_{\varepsilon}+w_{\varepsilon} \mathcal{I}_{\varepsilon}\left[w_{\varepsilon}\right] \Delta_{x} v_{\varepsilon} d x d y \\
\leq & \int_{\mathbb{R}^{M}} \int_{\mathbb{R}^{N}} \frac{w_{\varepsilon}^{2}}{2} \Delta_{x} \mathcal{I}_{\varepsilon}\left[u_{\varepsilon}\right]+2 w_{\varepsilon}^{2}+\frac{\left(\Delta_{x} \mathcal{I}_{\varepsilon}\left[w_{\varepsilon}\right] v_{\varepsilon}\right)^{2}}{2}+\left(\nabla_{x} \mathcal{I}_{\varepsilon}\left[w_{\varepsilon}\right] \cdot \nabla_{x} v_{\varepsilon}\right)^{2}+\frac{\left(\mathcal{I}_{\varepsilon}\left[w_{\varepsilon}\right] \Delta_{x} v_{\varepsilon}\right)^{2}}{2} d x d y \\
\leq & \left(\frac{\kappa_{0}}{2}+2+C \int_{\mathbb{R}^{M}} \int_{\mathbb{R}^{N}} \frac{v_{\varepsilon}^{2}}{2}+\left(\nabla_{x} v_{\varepsilon}\right)^{2}+\frac{\left(\Delta_{x} v_{\varepsilon}\right)^{2}}{2} d x d y\right) \int_{\mathbb{R}^{M}} \int_{\mathbb{R}^{N}} w_{\varepsilon}^{2} d x d y \\
\leq & \frac{\kappa_{5}}{2} e^{\kappa_{5} t} \int_{\mathbb{R}^{M}} \int_{\mathbb{R}^{N}} w_{\varepsilon}^{2} d x d y,
\end{aligned}
$$

for some constant $\kappa_{5}>0$ depending only on $b,\left\|u_{0}\right\|_{L^{1}\left(\mathbb{R}^{M} \times \mathbb{R}^{N}\right)}$, and $\left\|v_{0}\right\|_{L^{1}\left(\mathbb{R}^{M} \times \mathbb{R}^{N}\right)}$. Therefore, the Gronwall Lemma gives (2.4).

Proof of Theorem 2.2. This is now seen to be a direct consequence of Lemmas 2.10, 2.11, and 2.12.

We are now ready to prove Theorem 2.1.

Proof of Theorem 2.1. For every $0<\varepsilon<1$ let $u_{\varepsilon}$ be the solution of (2.5). For every $T>0$, Lemmas 2.4, 2.5, and 2.6 guarantee that

$$
\begin{aligned}
& \left\{u_{\varepsilon}\right\}_{\varepsilon} \text { is bounded in } L^{\infty}\left(0, T ; H^{1}\left(\mathbb{R}^{M} \times \mathbb{R}^{N}\right)\right), \\
& \left\{\partial_{t} u_{\varepsilon}\right\}_{\varepsilon} \text { is bounded in } L^{2}\left((0, T) \times \mathbb{R}^{M} \times \mathbb{R}^{N}\right),
\end{aligned}
$$

Since (see [8])

$$
H^{1}\left(\mathbb{R}^{M} \times \mathbb{R}^{N}\right) \subset \subset L_{\text {loc }}^{2}\left(\mathbb{R}^{M} \times \mathbb{R}^{N}\right),
$$

using [9, Theorem 5], there exists a subsequence $\left\{u_{\varepsilon_{k}}\right\}_{k}$ and a function $u$ such that

$$
u_{\varepsilon_{k}} \rightarrow u \quad \text { in } L_{\text {loc }}^{2}((0, T) \times \mathbb{R}) .
$$

Therefore, thanks to (2.29), we have

$$
u_{\varepsilon_{k}} \rightarrow u \quad \text { in } L^{p}\left(0, T ; L^{2}\left(\mathbb{R}^{M} \times \mathbb{R}^{N}\right)\right), 1 \leq p<\infty .
$$

Moreover, Lemmas 2.1, 2.2, 2.3, 2.4, 2.5, and 2.6 imply (D.1), (2.6), and (D.2). 
Finally, (2.3) follows from (2.26) and the same argument of (2.9) gives (2.4) and Lemma 2.11 proves (2.8).

\section{A Difference Method in $(1+1)$ D}

We present the scheme in the semi-discrete case where the $t$ variable is continuous. To this end, fix small positive $\Delta x$ and $\Delta y$, and set $x_{j}=j \Delta x, y_{k}=k \Delta y$ for integers $j$ and $k$. With the usual notation $u_{j k}(t) \approx u\left(t, x_{j}, y_{k}\right)$, define the discrete derivatives

$$
D_{ \pm}^{x} u_{j, k}= \pm \frac{u_{j \pm 1, k}-u_{j, k}}{\Delta x}
$$

The discrete derivatives $D_{+}^{y}, D_{-}^{y}$ and $D_{\mathrm{c}}^{y}$ are defined analogously. When possible, we shall use the abbreviated notation $u=\left\{u_{j k}\right\}_{j, k \in \mathbb{Z}}$, as well as $u=u_{j k}$, for any $j$ and $\mathrm{k}$, so that $\sum_{j k} u=\sum_{j k} u_{j k}$ etc.

The scheme reads

$$
\left\{\begin{aligned}
\partial_{t} u_{j k}= & I_{j} D_{+}^{x} D_{-}^{x} u_{j k}+2\left(D_{-}^{x} I_{j} \wedge 0\right) D_{-}^{x} u_{j k} & & \\
& +2\left(D_{+}^{x} I_{j} \vee 0\right) D_{+}^{x} u_{j k}+D_{+}^{x} D_{-}^{x} I_{j} u_{j k}+D_{+}^{y} D_{-}^{y} u_{j k}, & & t>0, j, k \\
u_{j k}(0)= & u_{0}\left(x_{j}, y_{k}\right) & & j, k
\end{aligned}\right.
$$

where the initial datum $u_{0}$ is assumed to be a continuous function defined on $\mathbb{R}^{2}$ and $(a \wedge 0)=$ $\min \{a, 0\},(a \vee 0)=\max \{a, 0\}$. In order to define $I_{i}$, we need to introduce the following piecewise constant function

where

$$
u_{\Delta x, \Delta y}(t, x, y)=u_{j k}(t), \quad(x, y) \in \mathcal{R}_{j, \Delta x}^{x} \times \mathcal{R}_{j, \Delta y}^{y},
$$

$$
\mathcal{R}_{j, \Delta x}^{x}=\left[\left(j-\frac{1}{2}\right) \Delta x,\left(j+\frac{1}{2}\right) \Delta x\right), \quad \mathcal{R}_{j, \Delta y}^{y}=\left[\left(k-\frac{1}{2}\right) \Delta y,\left(k+\frac{1}{2}\right) \Delta y\right) .
$$

We define $I_{j}$ as follows

$$
I_{j}(t)=I_{j}[u](t)=\mathcal{I}\left[u_{\Delta x, \Delta y}\right]\left(t, x_{j}\right)=\iint b\left(x_{j}, y\right) u_{\Delta x, \Delta y}(t, \xi, y) d \xi d y .
$$

To emphasize the dependence on the argument of $\mathcal{I}$, we also write

$$
L[v]=I[v] D_{+}^{x} D_{-}^{x}+2\left(D_{-}^{x} I[v] \wedge 0\right) D_{-}^{x}+2\left(D_{+}^{x} I[v] \vee 0\right) D_{+}^{x}+\left(D_{+}^{x} D_{-}^{x} I[v]\right)+D_{+}^{y} D_{-}^{y},
$$

where $v$ is some fixed nonnegative function in $L^{1}(\mathbb{R})$.

Regarding the solvability of (3.1), we can argue as in the continuous case, by first obtaining á priori estimates on the solution of

$$
\partial_{t} \tau(v)=L[v] \tau(v)
$$

for a fixed nonnegative function $v=v(t, x)$, and then showing that for small $t$, the mapping $\tau$ has a fixed point.

Introducing the following piecewise constant functions

$$
\begin{aligned}
& u_{0, \Delta x, \Delta y}(x, y)=u_{0}\left(x_{j}, y_{k}\right),(x, y) \in \mathcal{R}_{j, \Delta x}^{x} \times \mathcal{R}_{j, \Delta y}^{y}, \\
& I_{\Delta x}(t, x)=I_{i}(t), \quad x \in \mathcal{R}_{j, \Delta x}^{x},
\end{aligned}
$$

we can rewrite the scheme (3.1) in the following form

$$
\left\{\begin{array}{rlrl}
\partial_{t} u_{\Delta x, \Delta y}= & I_{\Delta x} D_{+}^{x} D_{-}^{x} u_{\Delta x, \Delta y} & & \\
& +2\left(D_{-}^{x} I_{\Delta x} \wedge 0\right) D_{-}^{x} u_{\Delta x, \Delta y}+2\left(D_{+}^{x} I_{\Delta x} \vee 0\right) D_{+}^{x} u_{\Delta x, \Delta y} & & \\
& +\left(D_{+}^{x} D_{-}^{x} I_{\Delta x}\right) u_{\Delta x, \Delta y}+D_{+}^{y} D_{-}^{y} u_{\Delta x, \Delta y}, & & t>0, x, y \in \mathbb{R} \\
u_{\Delta x, \Delta y}(0, x, y)=u_{0, \Delta x, \Delta y}(x, y), & x, y \in \mathbb{R} .
\end{array}\right.
$$

The main result of this section is the following. 
Theorem 3.1. Assume that (H.1), (H.2), and (2.2) hold. We have that

$$
\begin{aligned}
& u_{\Delta x, \Delta y} \rightarrow u \text { strongly in } L^{p}\left(0, T ; L^{2}\left(\mathbb{R}^{2}\right)\right), 1 \leq p<\infty, T>0, \text { as } \Delta x, \Delta y \rightarrow 0, \\
& \left\|u_{\Delta x, \Delta y}(t, \cdot, \cdot)-u(t, \cdot, \cdot)\right\|_{L^{2}\left(\mathbb{R}^{2}\right)} \leq k e^{k t}\left(\Delta x \Delta y+\left\|u_{0, \Delta x, \Delta y}-u_{0}\right\|_{L^{2}\left(\mathbb{R}^{2}\right)}\right), \quad t \geq 0
\end{aligned}
$$

for some constant $k$ depending only on $b$ and $u_{0}$, where $u$ is the unique solution of (1.1) with the initial datum $u_{0}$.

The following lemmas are needed.

Lemma 3.1 (Pointwise bounds). We have that

$$
0 \leq u_{\Delta x, \Delta y}(t, x, y) \leq e^{k t} u^{*}, \quad(t, x, y) \in[0, \infty) \times \mathbb{R}^{2},
$$

for some constant $k$ depending only on $b$ and $u_{0}$.

Proof. Assume that $u_{0}>0$ for all $j$, and that $\min _{i \ell} u_{u \ell}(t)=u_{j k}(t)=0$ for some $t>0$. Then $\partial_{t} u_{j k}(t) \leq 0$, if $t$ is such that $u_{i \ell}(s)>0$ for all $s<t$ and all $i$ and $\ell$. We may choose $j$ and $k$ such that if $D_{+}^{y} D_{-}^{y} u_{j k}=0$, then either $D_{+}^{x} u_{j k}>0$ and $D_{-}^{x} u_{j k} \leq 0$ or $D_{+}^{x} u_{j k} \geq 0$ and $D_{-}^{x} u_{j k}>0$. Or $D_{+}^{x} D_{-}^{x} u_{j k} \geq 0$ and $D_{+}^{y} D_{-}^{y} u_{i j}>0$. This is so since otherwise we would have $u_{j k}(t)=0$ for all $j, k$ and $u_{j k}(s)=0$ for all $s>t$ and all $j, k$. Hence $I_{j} D_{+}^{x} D_{-}^{x} u_{j}(t)+D_{+}^{y} D_{-}^{y} u_{j k}>0$. We get

$$
\begin{aligned}
0 & \geq \partial_{t} u_{j k}=I_{j} D_{+}^{x} D_{-}^{x} u_{j k}+2\left(D_{-}^{x} I_{j} \wedge 0\right) D_{-}^{x} u_{j k}+2\left(D_{+}^{x} I_{j} \vee 0\right) D_{+}^{x} u_{j k}+D_{+}^{y} D_{-}^{y} u_{j k} \\
& >I_{j} D_{+}^{x} D_{-}^{x} u_{j k}+D_{+}^{y} D_{-}^{y} u_{j k}>0,
\end{aligned}
$$

which is a contradiction. Hence $u>0$.

For an upper bound, set $w=e^{-k t} u$, we have that

$$
\partial_{t} w=I D_{+}^{x} D_{-}^{x} w+2\left(\left(D_{-}^{x} I \wedge 0\right) D_{-}^{x} w+\left(D_{+}^{x} I \vee 0\right) D_{+}^{x} w\right)+\left(D_{+}^{x} D_{-}^{x} I-k\right) w+D_{+}^{y} D_{-}^{y} w .
$$

We shall see later that $u \in L^{\infty}\left(0, \infty ; L^{1}\left(\mathbb{R}^{2}\right)\right)$, bounded independently of $t, \Delta x$ and $\Delta y$, hence $u_{j k}<\mathcal{O}\left(\|u\|_{L^{\infty}\left(L^{1}\right)} /(\Delta x \Delta y)\right)$. If $u$ has a local maximum we have that $D_{+}^{x} D_{-}^{x} u_{i j} \leq 0$ and $D_{+}^{y} D_{-}^{y} u_{i j} \leq 0$, with one inequality being strict, and $u_{i j}$ is the value of the local maximum.

$$
\begin{aligned}
\partial_{t} w_{i j} & =I_{i} D_{+}^{x} D_{-}^{x} w_{i j}+2\left(D_{-}^{x} I_{i} \wedge 0\right) D_{-}^{x} w_{i j}+2\left(D_{+}^{x} I_{i} \vee 0\right) D_{+}^{x} w_{i j}+\left(D_{+}^{x} D_{-}^{x} I_{i}-k\right) w_{i j}+D_{+}^{y} D_{-}^{y} w_{i j} \\
& <\left(D_{+}^{x} D_{-}^{x} I_{i}-k\right) w_{i j}<0,
\end{aligned}
$$

if we choose $k>\sup _{i} D_{+}^{x} D_{-}^{x} I_{i}$. Thus $w<\max _{i j} w_{i j}(0)$, and $u(t) \leq e^{k t} \max _{i j} u_{i j}(0)$.

Lemma 3.2 ( $L^{1}$ bound). We have that

$$
\left\|u_{\Delta x, \Delta y}(t, \cdot, \cdot)\right\|_{L^{1}\left(\mathbb{R}^{2}\right)}=\left\|u_{0}\right\|_{L^{1}\left(\mathbb{R}^{2}\right)},
$$

for every $t>0$.

Proof. We have that

$$
\begin{aligned}
& \frac{d}{d t}\left\|u_{\Delta x, \Delta y}(t, \cdot, \cdot)\right\|_{L^{1}\left(\mathbb{R}^{2}\right)}=\Delta x \Delta y \frac{d}{d t} \sum_{j k} u_{j k}=\Delta x \Delta y \sum_{j k} \partial_{t} u_{j k} \\
&= \Delta x \Delta y \sum_{j k}\left(I_{j} D_{+}^{x} D_{-}^{x} u_{j k}+2\left(D_{-}^{x} I_{j} \wedge 0\right) D_{-}^{x} u_{j k}\right. \\
&\left.+2\left(D_{+}^{x} I_{j} \vee 0\right) D_{+}^{x} u_{j k}+D_{+}^{x} D_{-}^{x} I_{j} u_{j k}+D_{+}^{y} D_{-}^{y} u_{j k}\right) \\
&= 2 \Delta x \Delta y \sum_{j k} \underbrace{\left(D_{+}^{x}\left(D_{-}^{x} I_{j} \wedge 0\right)+D_{-}^{x}\left(D_{+}^{x} I_{j} \vee 0\right)+D_{+}^{x} D_{-}^{x} I_{j}\right)}_{=0} u_{j k}=0 .
\end{aligned}
$$


Remark. Strictly speaking, the above computations assumed that $\lim _{i j \rightarrow \infty} u_{i j} D_{+}^{x} u_{i j}=0$, and a similar assumption must be imposed for the discrete $y$ derivative. However, our calculation can be made rigorous by multiplying the equation for $u_{t}$ by a smooth cut off function $\varphi_{\varepsilon}(x, y)$ such that

$$
\varphi_{\varepsilon}(x, y)= \begin{cases}1 & |x|+|y| \leq R_{\varepsilon}^{1}, \\ \in(0,1) & R_{\varepsilon}^{1}<|x|+|y|<R_{\varepsilon}^{2}, \\ 0 & |x|+|y| \geq R_{\varepsilon}^{2},\end{cases}
$$

We also have that $\lim _{\varepsilon \rightarrow 0} R_{\varepsilon}^{\ell}=\infty$ for $\ell=1,2$. This would lead to estimates that are $\varepsilon$ independent, and one would then let $\varepsilon$ tend to zero. In the subsequent computations we shall use summation by parts assuming that the relevant limits are zero without using this technique. Also these calculations can be justified rigorously by the same device.

Lemma 3.3 ( $L^{2}$ bound). We have that

$$
\left\|u_{\Delta x, \Delta y}(t, \cdot, \cdot)\right\|_{L^{2}\left(\mathbb{R}^{2}\right)} \leq\left\|u_{0}\right\|_{L^{2}\left(\mathbb{R}^{2}\right)} e^{k t},
$$

for some constant $k$ depending only on $\left\|u_{0}\right\|_{L^{1}(\mathbb{R})}$ and $\partial_{x}^{2} b$.

Proof. Since

We have that

$$
u D_{ \pm} u=\frac{1}{2} D_{ \pm} u^{2} \mp \frac{\Delta x}{2}\left(D_{ \pm} u\right)^{2}
$$

$$
\begin{aligned}
& \frac{1}{2} \frac{d}{d t}\left\|u_{\Delta x, \Delta y}(t, \cdot, \cdot)\right\|_{L^{2}\left(\mathbb{R}^{2}\right)}^{2}=\Delta x \Delta y \frac{d}{d t} \sum_{j k} \frac{u_{j k}^{2}}{2}=\Delta x \Delta y \sum_{j k} u_{j k} \partial_{t} u_{j k} \\
& =\Delta x \Delta y \sum_{j k}\left(u_{j k} I_{j} D_{+}^{x} D_{-}^{x} u_{j k}+2 u_{j k}\left(D_{-}^{x} I_{j} \wedge 0\right) D_{-}^{x} u_{j k}\right. \\
& \left.+2 u_{j k}\left(D_{+}^{x} I_{j} \vee 0\right) D_{+}^{x} u_{j k}+D_{+}^{x} D_{-}^{x} I_{j} u_{j k}^{2}+u_{j k} D_{+}^{y} D_{-}^{y} u_{j k}\right) \\
& =\Delta x \Delta y \sum_{j k}(\underbrace{-I_{j}\left(D_{-}^{x} u_{j k}\right)^{2}}_{\leq 0}-u_{j k} D_{-}^{x} I_{j} D_{-}^{x} u_{j k}+2 u_{j k}\left(D_{-}^{x} I_{j} \wedge 0\right) D_{-}^{x} u_{j k} \\
& +2 u_{j k}\left(D_{+}^{x} I_{j} \vee 0\right) D_{+}^{x} u_{j k}+D_{+}^{x} D_{-}^{x} I_{j} u_{j k}^{2} \underbrace{-\left(D_{-}^{y} u_{j k}\right)^{2}}_{\leq 0}) \\
& \leq \Delta x \Delta y \sum_{j k}(-\frac{D_{-}^{x} I_{j}}{2} D_{-}^{x}\left(u_{j k}\right)^{2} \underbrace{-\frac{\Delta x}{2} D_{-}^{x} I_{j}\left(D_{-}^{x} u\right)^{2}}_{\leq 0}+\left(D_{-}^{x} I_{j} \wedge 0\right) D_{-}^{x}\left(u_{j k}\right)^{2}) \\
& +\Delta x \Delta y \sum_{j k}\left(\left(D_{+}^{x} I_{j} \vee 0\right) D_{+}^{x}\left(u_{j k}\right)^{2}+D_{+}^{x} D_{-}^{x} I_{j} u_{j k}^{2}\right) \\
& +\Delta x \Delta y \underbrace{\sum_{j k}\left(\Delta x\left(D_{-}^{x} I_{j} \wedge 0\right)\left(D_{-}^{x} u\right)^{2}-\Delta x\left(D_{+}^{x} I_{j} \vee 0\right)\left(D_{+}^{x} u\right)^{2}\right)}_{=0} \\
& \leq \Delta x \Delta y \sum_{j k}\left(\frac{D_{+}^{x} D_{-}^{x} I_{j}}{2}-D_{+}^{x}\left(D_{-}^{x} I_{j} \wedge 0\right)-D_{-}^{x}\left(D_{+}^{x} I_{j} \vee 0\right)\right) u_{j k}^{2} \\
& \leq-\Delta x \Delta y \sum_{j k} \frac{D_{+}^{x} D_{-}^{x} I_{j}}{2} u_{j k}^{2} \leq C \Delta x \Delta y \sum_{j k} u_{j k}^{2},
\end{aligned}
$$

where $C$ is a constant depending only on $\partial_{x}^{2} b$ and $\left\|u_{0}\right\|_{L^{1}(\mathbb{R})}$. Therefore, the claim follows from the Gronwall Lemma. 
Lemma 3.4 ( $H^{1}$ bound). We have that

$$
\left\|D u_{\Delta x, \Delta y}(t, \cdot, \cdot)\right\|_{L^{2}\left(\mathbb{R}^{2}\right)} \leq e^{C t}\|D u(0)\|_{L^{2}\left(\mathbb{R}^{2}\right)}+C\|u(0)\|_{L^{2}\left(\mathbb{R}^{2}\right)}\left(e^{k t}-1\right),
$$

for some constants $C$ and $k$ depending only on $b$ and its $x$ derivatives, where

$$
D u=\left(D_{-}^{x} u, D_{-}^{y} u\right) \text {. }
$$

Proof. Let $w=D_{-}^{x} u$, then

$$
\begin{aligned}
\partial_{t} w=D_{-}^{x} & \left(I D_{+}^{x} w\right)+2\left(D_{-}^{x} I \wedge 0\right) D_{-}^{x} w+2 D_{-}^{x}\left(D_{-}^{x} I \wedge 0\right) w^{-} \\
& +2\left(D_{+}^{x} I \vee 0\right) D_{+}^{x} w+2 D_{-}^{x}\left(D_{+}^{x} I \vee 0\right) w+D_{-}^{x} D_{+}^{x} I w+D_{-}^{x} D_{-}^{x} D_{+}^{x} I u^{-}+D_{+}^{y} D_{-}^{y} w
\end{aligned}
$$

where $w_{i j}^{-}=w_{i-1, j}$ and $u_{i j}^{-}=u_{i-1, j}$. Therefore

$$
\begin{aligned}
\sum_{i j} w_{i j}^{2}= & \sum_{i j}-I\left(D_{+}^{x} w_{i j}\right)^{2}-\left(D_{-}^{y} w_{i j}\right)^{2}+\left(D_{-}^{x} I \wedge 0\right)\left(D_{-}^{x} w_{i j}^{2}+\Delta x\left(D_{-}^{x} w_{i j}\right)^{2}\right) \\
& +\left(D_{+}^{x} I \vee 0\right)\left(D_{+}^{x} w_{i j}^{2}-\Delta x\left(D_{+}^{x} w_{i j}\right)^{2}\right)+D_{-}^{x} D_{+}^{x} I w^{2}+\left(D_{-}^{x} D_{-}^{x} D_{+}^{x} I\right) u_{i-1, j} w \\
\leq & -\sum_{i j} I_{i j}\left(D_{+}^{x} w_{i j}\right)^{2}+\left(D_{-}^{y} w_{i j}\right)^{2}-\Delta x\left(\left(D_{-}^{x} I \wedge 0\right)\left(D_{-}^{x} w_{i j}\right)^{2}-\left(D_{+}^{x} I \vee 0\right)\left(D_{+}^{x} w_{i j}\right)^{2}\right) \\
& +C\left(\left\|b_{x x}\right\|_{L^{\infty}}+\left\|b_{x x x}\right\|_{L^{\infty}}\right) \sum_{i j} w_{i j}^{2}+u_{i j}^{2} .
\end{aligned}
$$

Since $I[u]$ is independent of $j$, we have that $\left(D_{-}^{y} u\right)_{t}=L[u]\left(D_{-}^{y} u\right)$. Therefore, using the same arguments as we used when obtaining the $L^{2}$ bound on $u$, we obtain the bound

$$
\sum_{i j}\left(D_{-}^{y} u_{i j}(t)\right)^{2} \leq e^{C t} \sum_{i j}\left(D_{-}^{y} u_{i j}(0)\right)^{2}
$$

Thus setting $D u=\left(D_{-}^{x} u, D_{-}^{y} u\right)$, we get

$$
\|D u\|_{L^{2}\left(\mathbb{R}^{2}\right)} \leq e^{C t}\|D u(0)\|_{L^{2}\left(\mathbb{R}^{2}\right)}+C\|u(0)\|_{L^{2}\left(\mathbb{R}^{2}\right)}\left(e^{k t}-1\right),
$$

for some constants $C$ and $k$ depending only on $b$ and its $x$ derivatives.

Lemma 3.5 ( $H^{2}$ bound). We have that

$$
\left\|D^{2} u_{\Delta x, \Delta y}(t, \cdot, \cdot)\right\|_{L^{2}\left(\mathbb{R}^{2}\right)} \leq\left\|D^{2} u(0)\right\|_{L^{2}\left(\mathbb{R}^{2}\right)} e^{C t}+\left(e^{C t}-1\right)\left(\|u(0)\|_{L^{2}\left(\mathbb{R}^{2}\right)}+\|D u(0)\|_{L^{2}\left(\mathbb{R}^{2}\right)}\right),
$$

for some constant $C$ depending only on $\left\|u_{0}\right\|_{L^{1}\left(\mathbb{R}^{2}\right)}$ and on $b$ and its $x$ derivatives, where $D^{2} u$ denotes the discrete Hessian of $u$, i.e.,

$$
D^{2} u_{i j}=\left(\begin{array}{cc}
D_{+}^{x} D_{-}^{x} u_{i j} & D_{-}^{x} D_{+}^{y} u_{i j} \\
D_{+}^{y} D_{-}^{x} u_{i j} & D_{+}^{y} D_{-}^{y} u_{i j}
\end{array}\right)
$$

Proof. Let $z^{x x}=D_{+}^{x} D_{-}^{x} u$, then

$$
\begin{aligned}
\partial_{t} z_{i j}^{x x}=D_{+} D_{-}\left(I z_{i j}^{x x}\right)+D_{+}[ & 2\left(D_{-}^{x} I \wedge 0\right) z_{i-1, j}^{x x}+2 D_{-}\left(D_{-}^{x} I \wedge 0\right) D_{-}^{x} u_{i-1, j} \\
& +2\left(D_{+}^{x} I \vee 0\right) z_{i j}^{x x}+2 D_{-}\left(D_{+}^{x} I \vee 0\right) D_{-}^{x} u_{i j} \\
& \left.+D_{+}^{x} D_{-}^{x} I D_{-}^{x} u_{i j}+D_{-}^{x} D_{+}^{x} D_{-}^{x} I u_{i-1, j}\right]+D_{+}^{y} D_{-}^{y} z_{i j}^{x x} \\
= & I_{i} D_{+}^{x} D_{-}^{x} z_{i j}^{x x}+D_{+}^{x} I_{i} D_{+}^{x} z_{i j}^{x x}+D_{-}^{x} I_{i} D_{-}^{x} z_{i j}^{x x}+2\left(D_{-}^{x} I \wedge 0\right) D_{-}^{x} z_{i j}^{x x}+2\left(D_{+}^{x} I \vee 0\right) D_{+}^{x} z_{i j}^{x x} \\
+ & D_{+}^{y} D_{-}^{y} z_{i j}^{x x}+\text { "lower order terms", }
\end{aligned}
$$

where by "lower order terms" we mean terms depending linearly on $I$ and its derivatives, and on $z^{x x}, D_{ \pm}^{x, y} u$ and $u$, but not on $D_{ \pm}^{x} z^{x x}$ or $D_{+}^{x} D_{-}^{x} z^{x x}$. Multiplying by $z^{x x}$ on both sides and summing over $i$ and $j$, we get

$$
\begin{aligned}
\frac{1}{2} \frac{d}{d t} \sum_{j}\left(z_{i j}^{x x}\right)^{2} \leq & \sum_{i j} D_{+}^{x} I_{i} z_{i j}^{x x} D_{+}^{x} z_{i j}^{x x}+D_{-}^{x} I_{i} z_{i j}^{x x} D_{-}^{x} z_{i j}^{x x} \\
& +2\left(D_{-}^{x} I \wedge 0\right) z_{i j}^{x x} D_{-} z_{i j}^{x x}+2\left(D_{+}^{x} I \vee 0\right) z_{i j}^{x x} D_{+}^{x} z_{i j}^{x x}+\mathcal{K}
\end{aligned}
$$




$$
=: \mathcal{L}+\mathcal{K},
$$

where

$$
\mathcal{K}=C \sum_{i j}\left(z_{i j}^{x x}\right)^{2}+\left(D_{-}^{x} u_{i j}\right)^{2}+\left(D_{-}^{y} u_{i j}\right)^{2}+\left(u_{i j}\right)^{2},
$$

for some constant depending on the $x$-derivatives of $b$. Estimating $\mathcal{L}$ we find that

$$
\begin{aligned}
& \mathcal{L}=\sum_{i j}\left(D_{-}^{x} I_{i}+2\left(D_{-}^{x} I \wedge 0\right)\right)\left(\frac{1}{2} D_{-}^{x}\left(z_{i j}^{x x}\right)^{2}+\frac{\Delta x}{2}\left(D_{-}^{x} z^{x x}\right)^{2}\right) \\
&+\left(D_{+}^{x} I_{i}+2\left(D_{+}^{x} I \vee 0\right)\right)\left(\frac{1}{2} D_{+}^{x}\left(z_{i j}^{x x}\right)^{2}-\frac{\Delta x}{2}\left(D_{+}^{x} z_{i j}^{x x}\right)^{2}\right) \\
&=-\sum_{i j} \frac{1}{2}\left(D_{+}^{x} D_{-}^{x} I_{i}+2 D_{+}^{x}\left(D_{-}^{x} I \wedge 0\right)+2 D_{-}^{x}\left(D_{+}^{x} I \vee 0\right)\right)\left(z_{i j}^{x x}\right)^{2} \\
& \quad+\sum_{i j} \frac{\Delta x}{2}\left(D_{-}^{x} z_{i j}^{x x}\right)^{2}\left(D_{-}^{x} I_{i}+2\left(D_{-}^{x} I \wedge 0\right)_{i}-D_{-}^{x} I_{i}-2\left(D_{+}^{x} I \vee 0\right)_{i-1}\right) \\
& \leq \sum_{i j} C\left(z_{i j}^{x x}\right)^{2} \\
& \sum_{i j} \Delta x\left(D_{-}^{x} z_{i j}^{x x}\right)^{2}\left(\left(D_{-}^{x} I \wedge 0\right)_{i}-\left(D_{+}^{x} I \vee 0\right)_{i}\right)+\Delta x\left(D_{-} z_{i j}^{x x}\right)^{2}\left(\left(D_{+}^{x} I \vee 0\right)_{i}-\left(D_{+}^{x} I \vee 0\right)_{i-1}\right) \\
&=C \sum_{i} I_{i}\left(z_{i j}^{x x}\right)^{2}-\sum I_{i j} \Delta x\left(D_{-}^{x} z^{x x}\right)^{2}\left|D_{-}^{x} I\right|+\Delta x^{2} D_{-}^{x}\left(D_{+}^{x} I \vee 0\right)_{i}\left(D_{-} z_{i j}^{x x}\right)^{2} \\
& \leq \sum_{i j} C\left(z_{i j}^{x x}\right)^{2}
\end{aligned}
$$

where we have used $\sum_{i j} \Delta x^{2}\left(D_{-}^{x} z_{i j}^{x x}\right)^{2} \leq 2 \sum_{i j}\left(z_{i j}^{x x}\right)^{2}$ in the last line. We conclude that

$$
\frac{d}{d t} \sum_{i j}\left(D_{+}^{x} D_{-}^{x} u_{i j}\right)^{2} \leq C \sum_{i j}\left(u_{i j}\right)^{2}+\left|D u_{i j}\right|^{2}+\left(D_{+}^{x} D_{-}^{x} u_{i j}\right)^{2} .
$$

for some constant $C$ only depending on $b$ and its derivatives.

Next, let $z^{x y}=D_{+}^{y} D_{-}^{x} u$. We have that

$$
\begin{aligned}
\partial_{t} z_{i j}^{x y}= & D_{-}^{x}\left(I_{i} D_{+}^{x} z_{i j}^{x y}\right)+2\left(\left(I_{x, i} \wedge 0\right)\right)\left(D_{-}^{x} z_{i j}^{x y}\right)+2\left(\left(I_{x, i} \vee 0\right)\right)\left(D_{+}^{x} z_{i j}^{x y}\right) \\
& +2\left(D_{-}^{x}\left(I_{x, i} \wedge 0\right)\right) z_{i-1, j}^{x y}+2\left(D_{-}^{x}\left(I_{x, i} \vee 0\right)\right) z_{i j}^{x y}+\left(D_{-}^{x} D_{+}^{x} D_{-}^{x} I_{i}\right)\left(D_{+}^{y} u_{i-1, j}\right)+D_{+}^{x} D_{-}^{x} I_{i} z_{i j}^{x y} \\
= & D_{-}^{x}\left(I_{i} D_{+}^{x} z_{i j}^{x y}\right)+2\left(\left(D_{-}^{x} I_{i} \wedge 0\right)\right)\left(D_{-}^{x} z_{i j}^{x y}\right)+2\left(\left(D_{+}^{x} I_{i} \vee 0\right)\right)\left(D_{+}^{x} z_{i j}^{x y}\right) \\
& \quad+\text { lower order terms, }
\end{aligned}
$$

where as before the "lower order terms" depend linearly on $D_{ \pm}^{x, y} u$, and on $z^{x y}$. Multiplying by $z^{x y}$ and summing over $i$ and $j$, we get

$$
\begin{aligned}
\frac{1}{2} \frac{d}{d t} \sum_{i j}\left(z_{i j}^{x y}\right)^{2} \leq & -\sum_{i j} I_{i j}\left(D_{+}^{x} z_{i j}^{x y}\right)^{2}+\left(D_{+}^{y} z_{i j}^{x y}\right)^{2} \\
& +2 \sum_{i j}\left(I_{x, i} \wedge 0\right) z_{i j}^{x y} D_{-}^{x} z_{i j}^{x y}+\left(I_{x, i} \vee 0\right) z_{i j}^{x y} D_{+}^{x} z_{i j}^{x y} \\
& +C \sum_{i j}\left(z_{i j}^{x y}\right)^{2}+\left|D u_{i j}\right|^{2} \\
\leq & \sum_{i j}\left(I_{x, i} \wedge 0\right)\left(D_{-}^{x}\left(z_{i j}^{x y}\right)^{2}+\Delta x\left(D_{-}^{x} z_{i j}^{x y}\right)^{2}\right)+\left(I_{x, i} \vee 0\right)\left(D_{+}^{x}\left(z_{i j}^{x y}\right)^{2}-\Delta x\left(D_{+}^{x} z_{i j}^{x y}\right)^{2}\right) \\
& +C \sum_{i j}\left(z_{i j}^{x y}\right)^{2}+\left|D u_{i j}\right|^{2} \\
\leq & C \sum_{i j}\left(z_{i j}^{x y}\right)^{2}+\left|D u_{i j}\right|^{2}
\end{aligned}
$$


Therefore

$$
\frac{d}{d t} \sum_{i j}\left(D_{+}^{y} D_{-}^{x} u_{i j}\right)^{2} \leq C \sum_{i j}\left(u_{i j}\right)^{2}+\left|D u_{i j}\right|^{2}+\left(D_{+}^{y} D_{-}^{x} u_{i j}\right)^{2} .
$$

To bound $D_{+}^{y} D_{-}^{y} u$, we note that

$$
\left(D_{+}^{y} D_{-}^{y} u\right)_{t}=L[u]\left(D_{+}^{y} D_{-}^{y} u\right),
$$

and then use the same techniques as used to obtain an $L^{2}$ bound on $u$ to conclude that

$$
\frac{d}{d t} \sum_{i j}\left(D_{+}^{y} D_{-}^{y} u_{i j}\right)^{2} \leq C \sum_{i j}\left(D_{+}^{y} D_{-}^{y} u_{i j}\right)^{2} .
$$

Equations (3.6), (3.7) and (3.8) imply that

$$
\left\|D^{2} u(t)\right\|_{L^{2}\left(\mathbb{R}^{2}\right)} \leq\left\|D^{2} u(0)\right\|_{L^{2}\left(\mathbb{R}^{2}\right)} e^{C t}+\left(e^{C t}-1\right)\left(\|u(0)\|_{L^{2}\left(\mathbb{R}^{2}\right)}+\|D u(0)\|_{L^{2}\left(\mathbb{R}^{2}\right)}\right),
$$

for some constant $C$ depending only on $\|u\|_{L^{1}\left(\mathbb{R}^{2}\right)}$ and on $b$ and its $x$ derivatives.

Proof of Theorem 3.1. The previous lemmas and the same argument of Lemma 2.10 and the uniqueness of $u$ give (3.4).

We have to prove (3.5). Consider the piecewise constant functions

$$
\begin{aligned}
& \quad \overline{u_{\Delta x, \Delta y}}(t, x, y)=u\left(t, x_{j}, y_{k}\right), \quad(x, y) \in \mathcal{R}_{j, \Delta x}^{x} \times \mathcal{R}_{j, \Delta y}^{y}, \\
& v(t)=u_{\Delta x, \Delta y}(t, \cdot, \cdot)-\overline{u_{\Delta x, \Delta y}}(t, \cdot, \cdot) .
\end{aligned}
$$

We have that

$$
\left\|u_{\Delta x, \Delta y}(t, \cdot, \cdot)-u(t, \cdot, \cdot)\right\|_{L^{2}\left(\mathbb{R}^{2}\right)} \leq \underbrace{\|v(t, \cdot, \cdot)\|_{L^{2}\left(\mathbb{R}^{2}\right)}}_{\mathcal{A}}+\underbrace{\left\|\overline{u_{\Delta x, \Delta y}}(t, \cdot, \cdot)-u(t, \cdot, \cdot)\right\|_{L^{2}\left(\mathbb{R}^{2}\right)}}_{\mathcal{B}} .
$$

The Hölder Inequality and (D.1) give

$$
\begin{aligned}
\mathcal{B}^{2} & =\sum_{j, k} \int_{\mathcal{R}_{j, \Delta x}^{x}} \int_{\mathcal{R}_{j, \Delta y}^{y}}\left|u(t, x, y)-u\left(t, x_{j}, y_{k}\right)\right|^{2} d x d y \\
& \leq \Delta x \Delta y \sum_{j, k}\left|\int_{\mathcal{R}_{j, \Delta x}^{x}} \int_{\mathcal{R}_{j, \Delta y}^{y}}\right| \nabla u(t, x, y)|d x d y|^{2} \\
& \leq(\Delta x \Delta y)^{2} \sum_{j, k} \int_{\mathcal{R}_{j, \Delta x}^{x}} \int_{\mathcal{R}_{j, \Delta y}^{y}}|\nabla u(t, x, y)|^{2} d x d y \\
& =(\Delta x \Delta y)^{2}\|\nabla u(t, \cdot, \cdot)\|_{L^{2}\left(\mathbb{R}^{2}\right)}^{2} \leq C(\Delta x \Delta y)^{2},
\end{aligned}
$$

therefore

$$
\mathcal{B} \leq C \Delta x \Delta y,
$$

for some constant $C$ depending only on $b$ and $u_{0}$.

On the other hand,

$$
\partial_{t} v_{j k}=\mathcal{C}_{j k}+\mathcal{D}_{j k}+\mathcal{E}_{j k}+\mathcal{F}_{j k},
$$

where

$$
\begin{aligned}
\mathcal{C}_{j k} & =I_{j} D_{+}^{x} D_{-}^{x} v_{j k}+2\left(D_{-}^{x} I_{j} \wedge 0\right) D_{-}^{x} v_{j k}+2\left(D_{+}^{x} I_{j} \vee 0\right) D_{+}^{x} v_{j k}+D_{+}^{x} D_{-}^{x} I_{j} v_{j k}+D_{+}^{y} D_{-}^{y} v_{j k}, \\
\mathcal{D}_{j k} & =I_{j} D_{+}^{x} D_{-}^{x} u\left(t, x_{j}, y_{k}\right)-\mathcal{I}\left(t, x_{j}\right) \partial_{x}^{2} u\left(t, x_{j}, y_{k}\right), \\
\mathcal{E}_{j k} & =2\left(D_{-}^{x} I_{j} \wedge 0\right) D_{-}^{x} u\left(t, x_{j}, y_{k}\right)+2\left(D_{+}^{x} I_{j} \vee 0\right) D_{+}^{x} u\left(t, x_{j}, y_{k}\right)-2 \partial_{x} \mathcal{I}\left(t, x_{j}\right) \partial_{x} u\left(t, x_{j}, y_{k}\right), \\
\mathcal{F}_{j k} & =\left(D_{+}^{x} D_{-}^{x} I_{j}-\partial_{x}^{2} \mathcal{I}\left(t, x_{j}\right)\right) u\left(t, x_{j}, y_{k}\right) .
\end{aligned}
$$

Arguing as in Lemma 3.3 we have

$$
\Delta x \Delta y \sum_{j, k} v_{j k}(t) \mathcal{C}_{j k} \leq k \Delta x \Delta y \sum_{j k} v_{j k}^{2}(t),
$$


for some constant $k>0$ depending only on $b$ and $u_{0}$.

We have that

$$
\begin{aligned}
& \Delta x \Delta y \sum_{j, k} v_{j k}(t) \mathcal{D}_{j k} \\
& \leq C \Delta x \Delta y \sum_{j, k} v_{j k}^{2}(t)+C \Delta x \Delta y \sum_{j, k}\left(I_{j}-\mathcal{I}\left(t, x_{j}\right)\right)^{2}\left(\partial_{x} D_{-}^{x} u\left(t, x_{j}, y_{k}\right)\right)^{2} \\
&+C \Delta x \Delta y \sum_{j, k}\left(\mathcal{I}\left(t, x_{j}\right)\right)^{2}\left(\partial_{x} D_{-}^{x} u\left(t, x_{j}, y_{k}\right)-\partial_{x}^{2} u\left(t, x_{j}, y_{k}\right)\right)^{2} \\
& \leq C \Delta x \Delta y \sum_{j, k} v_{j k}^{2}(t)+C \Delta x \Delta y \sup _{j}\left(I_{j}-\mathcal{I}\left(t, x_{j}\right)\right)^{2} \sum_{j, k}\left(D_{+}^{x} D_{-}^{x} u\left(t, x_{j}, y_{k}\right)\right)^{2} \\
&+C \Delta x \Delta y \sum_{j, k}\left(\mathcal{I}\left(t, x_{j}\right)\right)^{2}\left(D_{+}^{x} D_{-}^{x} u\left(t, x_{j}, y_{k}\right)-\partial_{x}^{2} u\left(t, x_{j}, y_{k}\right)\right)^{2} \\
& \leq C \Delta x \Delta y \sum_{j, k} v_{j k}^{2}(t)+C(\Delta x \Delta y)^{2} \int_{\mathbb{R}^{2}} \mathcal{I}[u]\left|D_{x, y}^{3} u\right|^{2} d y d x
\end{aligned}
$$

therefore

$$
\Delta x \Delta y \sum_{j, k} v_{j k}(t) \mathcal{D}_{j k} \leq C \Delta x \Delta y \sum_{j, k} v_{j k}^{2}(t)+C(\Delta x \Delta y)^{2} \int_{\mathbb{R}^{2}} \mathcal{I}[u]\left|D_{x, y}^{3} u\right|^{2} d y d x,
$$

for some constant $C>0$ depending only on $b$ and $u_{0}$.

Moreover

$$
\begin{aligned}
\Delta x \Delta y & \sum_{j, k} v_{j k}(t) \mathcal{E}_{j k} \\
\leq & C \Delta x \Delta y \sum_{j, k} v_{j k}^{2}(t)+C \Delta x \Delta y \sum_{j, k}\left(D_{-}^{x} I_{j}-\partial_{x} \mathcal{I}\left(t, x_{j}\right)\right)^{2}\left(D_{-}^{x} u\left(t, x_{j}, y_{k}\right)\right)^{2} \\
& +C \Delta x \Delta y \sum_{j, k}\left(\partial_{x} \mathcal{I}\left(t, x_{j}\right)\right)^{2}\left(D_{-}^{x} u\left(t, x_{j}, y_{k}\right)-\partial_{x} u\left(t, x_{j}, y_{k}\right)\right)^{2} \\
\leq & C \Delta x \Delta y \sum_{j, k} v_{j k}^{2}(t)+C \Delta x \Delta y \sup _{j}\left(D_{-}^{x} I_{j}-\partial_{x} \mathcal{I}\left(t, x_{j}\right)\right)^{2} \sum_{j, k}\left(D_{-}^{x} u\left(t, x_{j}, y_{k}\right)\right)^{2} \\
& +C \Delta x \Delta y \sup _{j}\left(\partial_{x} \mathcal{I}\left(t, x_{j}\right)\right)^{2} \sum_{j, k}\left(D_{-}^{x} u\left(t, x_{j}, y_{k}\right)-\partial_{x} u\left(t, x_{j}, y_{k}\right)\right)^{2} \\
\leq & C \Delta x \Delta y \sum_{j, k} v_{j k}^{2}(t)+C(\Delta x \Delta y)^{2}\|u(t, \cdot, \cdot)\|_{H^{2}\left(\mathbb{R}^{2}\right)}^{2}
\end{aligned}
$$

therefore

$$
\Delta x \Delta y \sum_{j, k} v_{j k}(t) \mathcal{E}_{j k} \leq C \Delta x \Delta y \sum_{j, k} v_{j k}^{2}(t)+C(\Delta x \Delta y)^{2},
$$

for some constant $C>0$ depending only on $b$ and $u_{0}$.

Since

$\Delta x \Delta y \sum_{j, k} v_{j k}(t) \mathcal{F}_{j k} \leq k \Delta x \Delta y \sum_{j, k} v_{j k}(t)^{2}+k \sup _{j}\left|D_{+}^{x} D_{-}^{x} I_{j}-\partial_{x}^{2} \mathcal{I}\left(t, x_{j}\right)\right|^{2} \Delta x \Delta y \sum_{j, k} u^{2}\left(t, x_{j}, y_{k}\right)$,

(D.1) gives

$$
\Delta x \Delta y \sum_{j, k} v_{j k}(t) \mathcal{F}_{j k} \leq k \sum_{j k} v_{j k}^{2}(t) .
$$

for some constant $k>0$ depending only on $b$ and $u_{0}$.

(3.13), (3.14), (3.15), and (3.16) guarantee

$$
\frac{d}{d t} \frac{\mathcal{A}^{2}}{2}=\frac{d}{d t} \frac{\Delta x \Delta y}{2} \sum_{j, k}\left|v_{j k}(t)\right|^{2}=\Delta x \Delta y \sum_{j, k} v_{j k}(t) \partial_{t} v_{j k}(t)
$$




$$
\begin{aligned}
= & \Delta x \Delta y \sum_{j, k} v_{j k}(t) \mathcal{C}_{j k}+\Delta x \Delta y \sum_{j, k} v_{j k}(t) \mathcal{D}_{j k} \\
& +\Delta x \Delta y \sum_{j, k} v_{j k}(t) \mathcal{E}_{j k}+\Delta x \Delta y \sum_{j, k} v_{j k}(t) \mathcal{F}_{j k} \\
\leq & C \Delta x \Delta y \sum_{j, k} v_{j k}^{2}(t)+C(\Delta x \Delta y)^{2}+C(\Delta x \Delta y)^{2} \int_{\mathbb{R}^{2}} \mathcal{I}[u]\left|D_{x, y}^{3} u\right|^{2} d y d x .
\end{aligned}
$$

Therefore, the Gronwall Lemma and Lemma 2.11 give

$$
\begin{aligned}
\mathcal{A}^{2}(t) \leq & e^{C t} \mathcal{A}^{2}(0)+C(\Delta x \Delta y)^{2}\left(e^{C t}-1\right) \\
& +C(\Delta x \Delta y)^{2} e^{C t} \int_{0}^{t} \int_{\mathbb{R}^{2}} e^{-C s} \mathcal{I}[u]\left|D_{x, y}^{3} u\right|^{2} d s d y d x \\
\leq & e^{C t} \mathcal{A}^{2}(0)+C(\Delta x \Delta y)^{2}\left(e^{C t}-1\right) \\
& +C(\Delta x \Delta y)^{2} e^{C t} \int_{0}^{t} \int_{\mathbb{R}^{2}} \mathcal{I}[u]\left|D_{x, y}^{3} u\right|^{2} d s d y d x \\
\leq & e^{C t} \mathcal{A}^{2}(0)+C(\Delta x \Delta y)^{2}\left(e^{C t}-1\right) \\
& +C(\Delta x \Delta y)^{2} e^{C t}\left\|u_{0}\right\|_{H^{2}\left(\mathbb{R}^{2}\right)}^{2}
\end{aligned}
$$

that gives

$$
\mathcal{A}(t) \leq e^{C t} \mathcal{A}(0)+C \Delta x \Delta y e^{C t},
$$

for some constant $C>0$ depending only on $b$ and $u_{0}$.

Clearly, (3.5) follows from (3.10), (3.11), and (3.17).

\section{Numerical EXPERIMENTS}

In order to calculate, we have to discretize (3.1) in time. Since the solution is sufficiently regular, we can achieve second order accuracy in time by using the Crank-Nicholson scheme below, (4.1). We must also consider a finite domain, and choose a square $(x, y) \in[-X, X] \times[-X, X]$ where $X$ is a fixed positive number.

With the notation in (3.2) we then define

$$
\frac{u_{j k}^{n+1}-u_{j k}^{n}}{\Delta t}=\frac{1}{2}\left(L\left[u^{n}\right] u^{n}+L\left[u^{n+1}\right] u^{n+1}\right)_{j k} .
$$

This is supposed to hold for $n \geq 0,0<j<N+1$ and $0<k<M+1$ where $(N+1) \Delta x=$ $(M+1) \Delta y=2 X$. For simplicity we impose Dirichlet boundary conditions

$$
u_{0, k}^{n}=u_{N+1, k}^{n}=u_{j, 0}^{n}=u_{j, M+1}^{n}=0,
$$

for $0<j<N+1$ and $0<k<M+1$ and all $n \geq 0$. To solve the nonlinear equation (4.1) for $u^{n+1}$ we used simple iteration, which worked well, and usually converged within a relative error of $10^{-4} \Delta x$ after $2-3$ iterations.

In our example, we choose the function $b$ as

$$
b(x, \xi)=\frac{1}{\delta \sqrt{\pi}} e^{-(x-\xi)^{2} / \delta^{2}}, \quad \delta=0.1 .
$$

We choose $X=1$ and initial data

$$
u_{0}(x, y)=16\left(1-x^{2}\right)\left(1-y^{2}\right) e^{-8\left(x^{2}+y^{2}\right)} .
$$

In Figure 1 we show the approximate solution using 300 grid points in each direction, and $\Delta t=$ $0.01 \Delta x$ for $t=0, t=0.03$ and $t=0.06$. In order to test the convergence of the scheme we computed the $L^{2}$ error for various grid sizes. The $L^{2}$ error is defined by

$$
\text { error }=100 \times \frac{\left\|u_{\Delta x, \Delta y}(t, \cdot, \cdot)-u_{\text {ref }}(t, \cdot, \cdot)\right\|_{L^{2}}}{\left\|u_{\text {ref }}(t, \cdot \cdot \cdot)\right\|_{L^{2}}},
$$



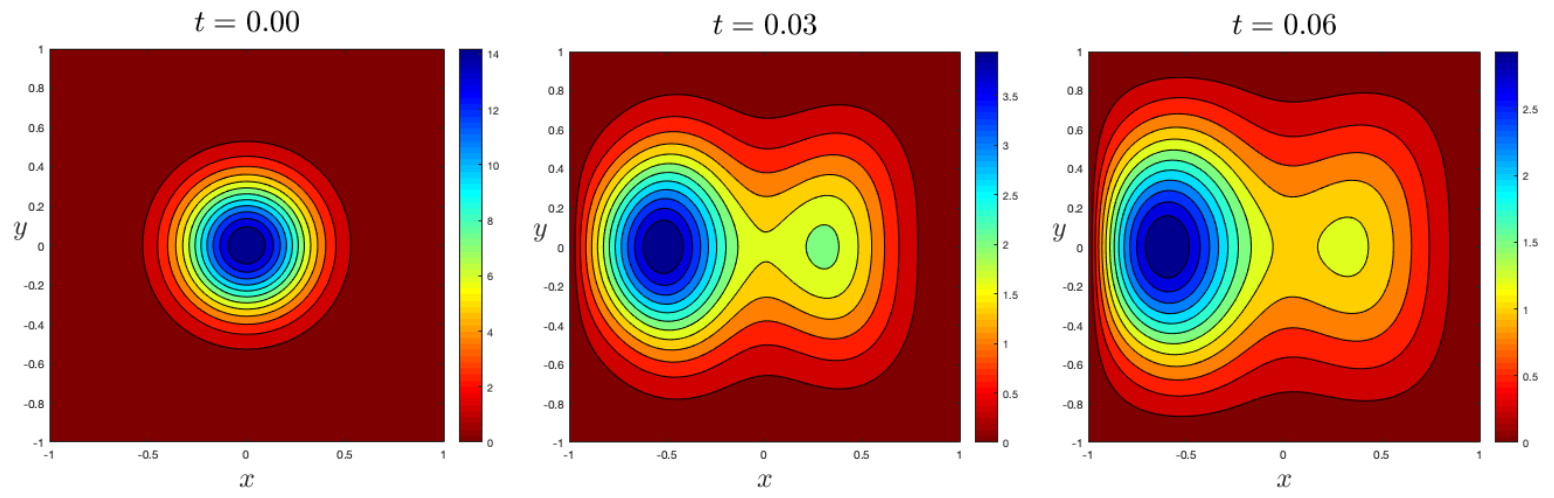

Figure 1. The approximate solution defined by (4.1) with initial data (4.4).

where $u_{\text {ref }}$ is a reference solution. In all of our computations we used $\Delta x=\Delta y=2 /(N+1)$, $t=0.06$ and the reference solution is the approximate solution computed by the scheme with $N=1600$. In Table 1 we show the errors and the convergence rate for various $N$. We see that the error seems to be first order in $\Delta x$, which is to be expected since the $L^{2}$ error in the approximation of the initial data by a piecewise constant function is first order, cf. (3.5).

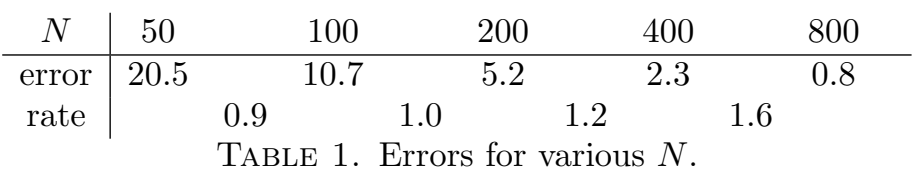

We remark that since we do not have any solution in closed form, the convergence we are measuring is only "self-convergence", and that actual convergence rates may be lower.

\section{REFERENCES}

[1] F. Abergel and R. Tachet, A nonlinear partial integro-differential equation from mathematical finance, Discrete Contin. Dyn. Syst. 27 (2010), no. 3, 907-917. MR 2629564

[2] F. Black and M. Scholes, The effects of dividend yield and dividend policy on common stock prices and returns, Journal of financial economics 1 (1974), no. 1, 1-22.

[3] G. M. Coclite, O. Reichmann, and N. H. Risebro, A convergent difference scheme for a class of partial integrodifferential equations modeling pricing under uncertainty, SIAM J. Numer. Anal. 54 (2016), no. 2, 588-605. MR 3472013

[4] J. Guyon and P. Henry-Labordere, The smile calibration problem solved, Available at SSRN 1885032 (2011).

[5] P. Henry-Labordere, Calibration of local stochastic volatility models to market smiles: A monte-carlo approach, Risk Magazine, September (2009).

[6] S. L. Heston, A closed-form solution for options with stochastic volatility with applications to bond and currency options, Review of financial studies 6 (1993), no. 2, 327-343.

[7] J. Hull and A. White, The general Hull-White model and supercalibration, Financial Analysts Journal $\mathbf{5 7}$ (2001), no. 6, 34-43.

[8] E. H. Lieb and M. Loss, Analysis, second ed., Graduate Studies in Mathematics, vol. 14, American Mathematical Society, Providence, RI, 2001. MR 1817225

[9] J. Simon, Compact sets in the space $L^{p}(0, T ; B)$, Ann. Mat. Pura Appl. (4) 146 (1987), 65-96. MR 916688

[10] A.-S. Sznitman, Topics in propagation of chaos, Ecole d'été de probabilités de Saint-Flour XIX1989, Springer, 1991, pp. 165-251.

(Giuseppe Maria Coclite)

Department of Mechanics, Mathematics and Management, Polytechnic University of Bari,

Via E. Orabona 4, I-70125 Bari, Italy

E-mail address: giuseppemaria.coclite@poliba.it

(Nils Henrik Risebro)

Department of Mathematics, University of Oslo, P.O. Box 1053, Blindern, N-0316 Oslo, Norway E-mail address: nilshr@math.uio.no 